\title{
Asymmetric Total Synthesis of the Naturally Occurring Antibiotic Anthracimycin
}

\author{
Emma K. Davison, ${ }^{\text {a,b,c }}$ Jared L. Freeman, ${ }^{\text {a,c }}$ Wanli Zhang, ${ }^{\text {d }}$ William M. Wuest, ${ }^{\text {dee }}$ Daniel P. \\ Furkert, ${ }^{\mathrm{a}, \mathrm{c}}$ and Margaret A. Brimble ${ }^{\mathrm{a}, \mathrm{b}, \mathrm{c}^{*}}$ \\ aSchool of Chemical Sciences, University of Auckland, 23 Symonds St., Auckland, 1010, New Zealand \\ ${ }^{b}$ School of Biological Sciences, University of Auckland, 3 Symonds St., Auckland, 1010, New Zealand \\ ${ }^{c}$ The Maurice Wilkins Centre for Molecular Biodiscovery, The University of Auckland, Private Bag 92019, \\ Auckland, 1142, New Zealand \\ ${ }^{d}$ Department of Chemistry, Emory University, 1515 Dickey Dr., Atlanta, GA 30322, USA \\ ${ }^{e}$ Emory Antibiotic Resistance Center, Emory School of Medicine, 201 Dowman Dr., Atlanta, GA 30322, USA
}

\section{SUPPORTING INFORMATION}


Wittig Reaction Optimisation $\quad$ S3

General Experimental Details $\quad$ S4

$\begin{array}{lr}\text { Experimental Procedures } & \text { S5-14 }\end{array}$

Table S2 ${ }^{1}$ H NMR comparison of natural (-)-1 with synthetic (-)-1 $\quad$ S15

Table S3 ${ }^{13}$ C NMR comparison of natural (-)-1 with synthetic (-)-1 S16

$\begin{array}{ll}\text { References } & \text { S17 }\end{array}$

${ }^{1} \mathrm{H}$ NMR spectrum of $\mathbf{5} \quad$ S18

${ }^{1} \mathrm{H}$ NMR spectrum of $\mathbf{3 a}$ and $\mathbf{3 b}(7: 1) \quad \mathrm{S} 19$

${ }^{13} \mathrm{C}$ NMR spectrum of $\mathbf{3 a}$ and $\mathbf{3 b}(7: 1) \quad$ S20

${ }^{1} \mathrm{H}$ NMR spectrum of $\mathbf{3 c} \quad$ S21

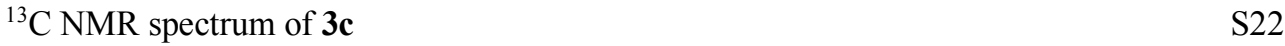

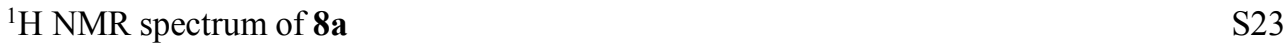

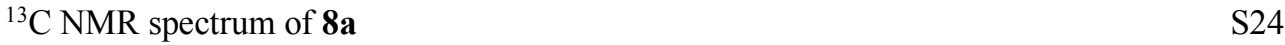

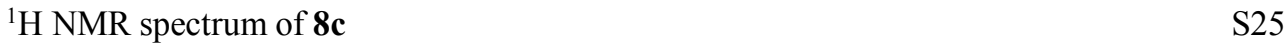

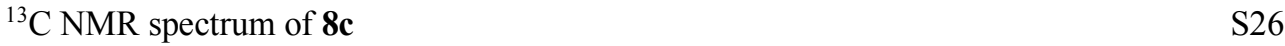

$\begin{array}{ll}\text { NOESY NMR spectrum of } 8 \mathbf{c} & \text { S27 }\end{array}$

$\begin{array}{ll}{ }^{1} \mathrm{H} \text { NMR spectrum of } 9 & \text { S28 }\end{array}$

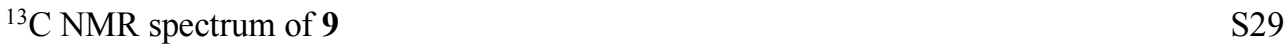

${ }^{1} \mathrm{H}$ NMR spectrum of $\mathbf{1 6} \quad \mathrm{S} 30$

${ }^{13} \mathrm{C}$ NMR spectrum of $\mathbf{1 6} \quad \mathrm{S} 31$

${ }^{1} \mathrm{H}$ NMR spectrum of $\mathbf{1 8} \quad$ S32

${ }^{13} \mathrm{C}$ NMR spectrum of $\mathbf{1 8} \quad$ S33

${ }^{1} \mathrm{H}$ NMR spectrum of $\mathbf{1 9} \quad \mathrm{S} 34$

${ }^{13} \mathrm{C}$ NMR spectrum of $\mathbf{1 9} \quad$ S35

${ }^{1} \mathrm{H}$ NMR spectrum of $\mathbf{2 0} \quad$ S36

$\begin{array}{ll}{ }^{13} \mathrm{C} \text { NMR spectrum of } \mathbf{2 0} & \text { S37 }\end{array}$

${ }^{1} \mathrm{H}$ NMR spectrum of $\mathbf{1 0} \quad \mathrm{S} 38$

$\begin{array}{lr}{ }^{13} \mathrm{C} \text { NMR spectrum of } \mathbf{1 0} & \text { S39 }\end{array}$

${ }^{1} \mathrm{H}$ NMR spectrum of $\mathbf{1 1} \quad \mathrm{S} 40$

${ }^{13} \mathrm{C}$ NMR spectrum of $\mathbf{1 1} \quad \mathrm{S} 41$

${ }^{1} \mathrm{H}$ NMR spectrum of $\mathbf{2 1} \quad \mathrm{S} 42$

${ }^{13} \mathrm{C}$ NMR spectrum of $\mathbf{2 1} \quad \mathrm{S} 43$

${ }^{1} \mathrm{H}$ NMR spectrum of crude (-)-1 and 2-epi-1 (1:1) S44

${ }^{1} \mathrm{H}$ NMR spectrum of (-)-1 $\quad$ S45

${ }^{13} \mathrm{C}$ NMR spectrum of $(-)-1 \quad$ S46 


\section{Wittig Reaction Optimisation}

Since Z-5 was previously found to be unreactive in the IMDA reaction, ${ }^{1}$ it was thought that improving the stereoselectivity of the Wittig reaction would ultimately result in improved access to IMDA adduct 3a. As such, Wittig reaction conditions were screened, as outlined in Table 1, and compared with the previously reported results (entry 1). ${ }^{1} \mathrm{~N}, \mathrm{~N}$-Dimethylaminopyridine (DMAP) has been reported to promote in situ olefin isomerisation in Wittig reactions with phosphonium salt $\mathbf{6}$, to selectively provide $(E)$ - $\alpha, \beta$-unsaturated imides. ${ }^{2}$ Unfortunately, addition of three equivalents of DMAP to the Wittig reaction of 6 with aldehyde 7 resulted in degradation (entry 2). However, catalytic $\mathrm{DMAP}^{3}$ resulted in a slightly improved $E / Z$ ratio of 5 (3:1), though with poor yield (27\% from alcohol 4, entry 3). Heating a mixture of 6 and 7 under reflux in dichloromethane, pleasingly afforded 5 in $65 \%$ yield, albeit with reduced stereoselectivity $(6: 5, E: Z)$ (entry 4$)$. Further increase in the reaction temperature $\left(80^{\circ} \mathrm{C}\right.$, toluene) resulted only in degradation (entry 5$)$. The effect of lithium salts was explored, and while this had no effect on the stereoselectivity of the reaction, the yield of $\mathbf{5}$ was improved to $81 \%$, with the additional benefit of reduced reaction time (entry 6). Pleasingly, the procedure was found to be amenable to gram scale $[1.7 \mathrm{~g}(5.15 \mathrm{mmol})$ of alcohol 4], yielding 5 in $62 \%$ yield over two steps $(1: 1, E: Z)$ (entry 7).

\section{Table S1. Optimisation of the Wittig reaction of 7 with 6}

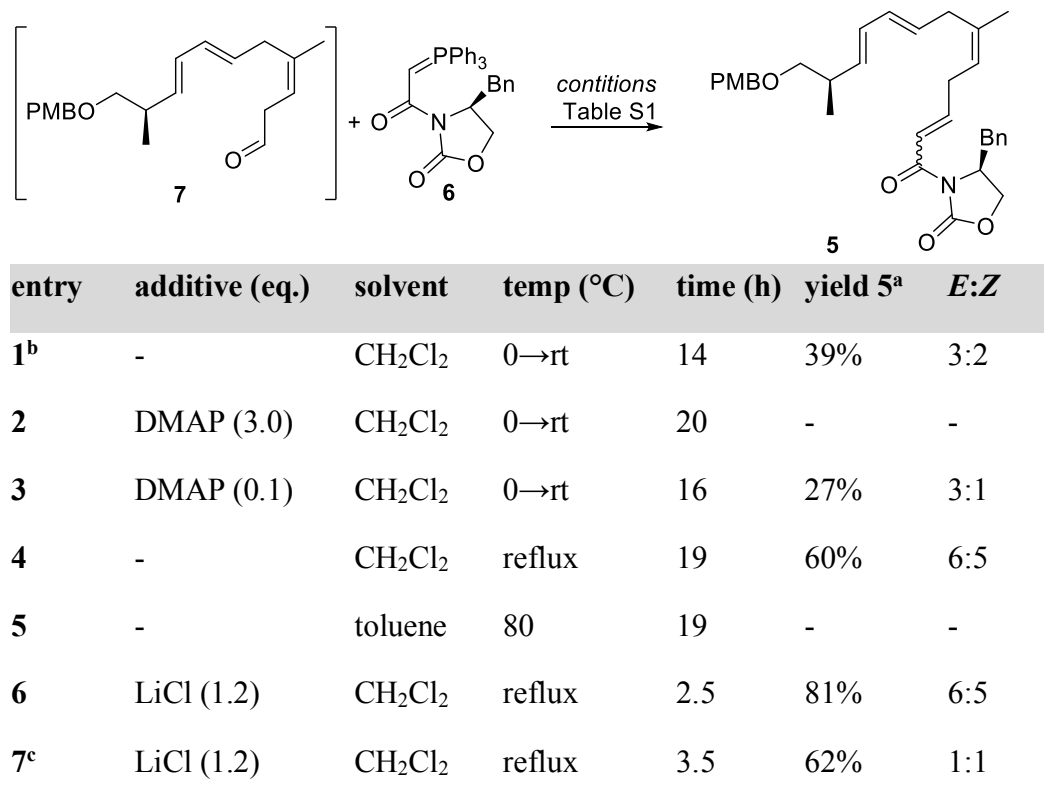

Reaction conducted on $0.071-0.212 \mathrm{mmol}$ scale unless otherwise stated. ${ }^{\text {a }}$ Yield over 2 steps from alcohol 4; ${ }^{b}$ previously established results for comparison ${ }^{1}$; ${ }^{\mathrm{c}} 5.15 \mathrm{mmol}$ scale 


\section{General Experimental Details}

Commercially available reagents were used throughout without purification unless otherwise stated. Anhydrous solvents were used as supplied. Diethyl ether, tetrahydrofuran, dichloromethane, dimethylformamide and toluene were dried using an LC Technology Solutions Inc. SP-1 solvent purification system under an atmosphere of dry nitrogen. Ether refers to diethyl ether. All reactions were routinely carried out in oven-dried glassware under a nitrogen atmosphere unless otherwise stated. All reactions requiring heating were done so using IKA magnetic hotplates with integrated temperature control sensors and DrySyn ${ }^{\circledR}$ heating blocks unless otherwise stated. Analytical thin layer chromatography was performed using silica plates and compounds were visualized at 254 and/or $365 \mathrm{~nm}$ ultraviolet irradiation followed by staining with either alkaline permanganate or ethanolic vanillin solution. Infrared spectra were obtained using a Perkin Elmer spectrum One Fourier Transform Infrared spectrometer. Absorption maxima are expressed in wavenumbers $\left(\mathrm{cm}^{-1}\right)$. Optical rotations were determined at the sodium D line $(589 \mathrm{~nm})$ at $23{ }^{\circ} \mathrm{C}$ by using an Autopol IV instrument, with concentrations given in $\mathrm{g} / 100 \mathrm{~mL}$. NMR spectra were recorded as indicated on an NMR spectrometer operating at $400 \mathrm{MHz}$ or $500 \mathrm{MHz}$ for ${ }^{1} \mathrm{H}$ nuclei and $100 \mathrm{MHz}$ or $125 \mathrm{MHz}$ for ${ }^{13} \mathrm{C}$ nuclei as specified. Chemical shifts are reported in parts per million $(\mathrm{ppm})$ relative to the chloroform $(\delta 7.26 \mathrm{ppm})$ peak. The ${ }^{13} \mathrm{C}$ NMR values were referenced to the residual chloroform peak. ${ }^{13} \mathrm{C}$ NMR values are reported as chemical shift $\delta$ and assignment. ${ }^{1} \mathrm{H}$ NMR shift values are reported as chemical shift $\delta$, relative integral, multiplicity (s, singlet; d, doublet; t, triplet; q, quartet; pent, pentet; m, multiplet; br, broad; app., apparent), coupling constant ( $\mathrm{J}$ in $\mathrm{Hz}$ ) and assignment. Assignments are made with the aid of DEPT 90, DEPT 135, COSY, NOESY and HSQC experiments. All experiments were conducted at $298 \mathrm{~K}$. Conventional NMR tubes ( $5 \mathrm{~mm}$ diameter, Wilmad) using a sample volume of $500 \mu \mathrm{L}$ were used, except for the spectra obtained for antracimycin (1) which employed a shigemi NMR tube using a sample volume of $150 \mu \mathrm{L}$. High resolution mass spectra were obtained by electrospray ionization in positive ion mode at a nominal accelerating voltage of $70 \mathrm{eV}$ on a microTOF mass spectrometer. 


\section{Experimental Procedures}

Tetraene 5

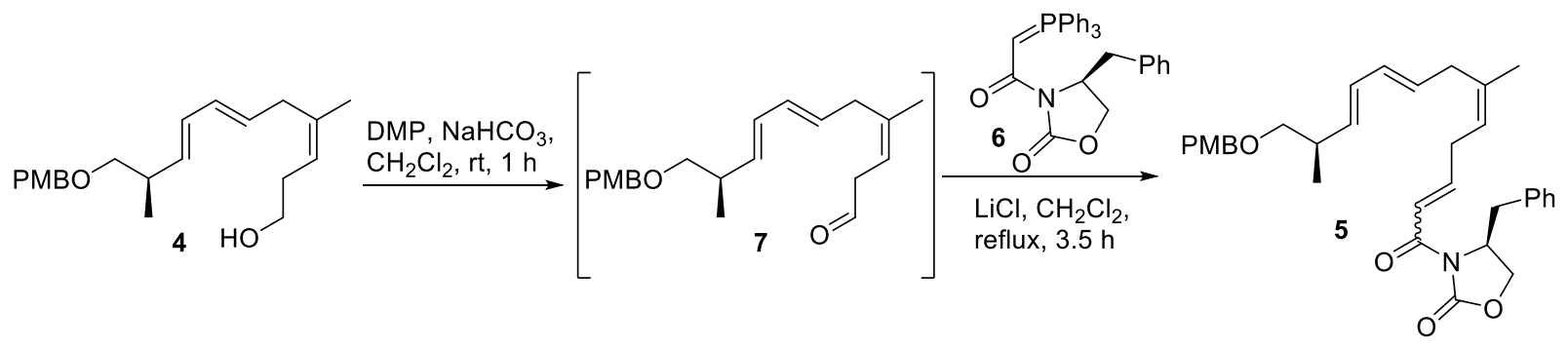

Aldehyde 7 was prepared from alcohol $4(1.69 \mathrm{~g}, 5.12 \mathrm{mmol})$ according to the procedure described by Brimble and coworkers. ${ }^{1}$ The crude aldehyde was then used directly in the next step without further purification due to its observed partial instability on silica gel. To a stirred solution of crude aldehyde 7 (assumed $5.12 \mathrm{mmol}$ ) in dichloromethane $(100 \mathrm{~mL})$ was added phosphorane $6^{4}(6.24 \mathrm{~g}, 13.0 \mathrm{mmol})$ and lithium chloride $(265 \mathrm{mg}, 6.25$ mmol) and the resulting mixture was heated to reflux for $3.5 \mathrm{~h}$. The reaction mixture was then cooled to room temperature, and concentrated in vacuo. The crude product was purified by flash chromatography on silica gel, eluting with petroleum ether - ethyl acetate (3:1) to afford the title compound (1.67 g, 3.15 mmol, 62\%, 1:1 inseparable mixture of $E / Z$ isomers) as a yellow oil. HRMS (ESI) m/z: $[\mathrm{M}+\mathrm{Na}]^{+}$calcd for $\mathrm{C}_{33} \mathrm{H}_{39} \mathrm{NO}_{5} \mathrm{Na}$ 552.2720; found 552.2723. Spectroscopic data was concordant with the literature ${ }^{1}$. 
IMDA adduct $\mathbf{3 a}$ and diastereomers $\mathbf{3 b}$ and $\mathbf{3} \mathbf{c}^{\mathrm{a}}$

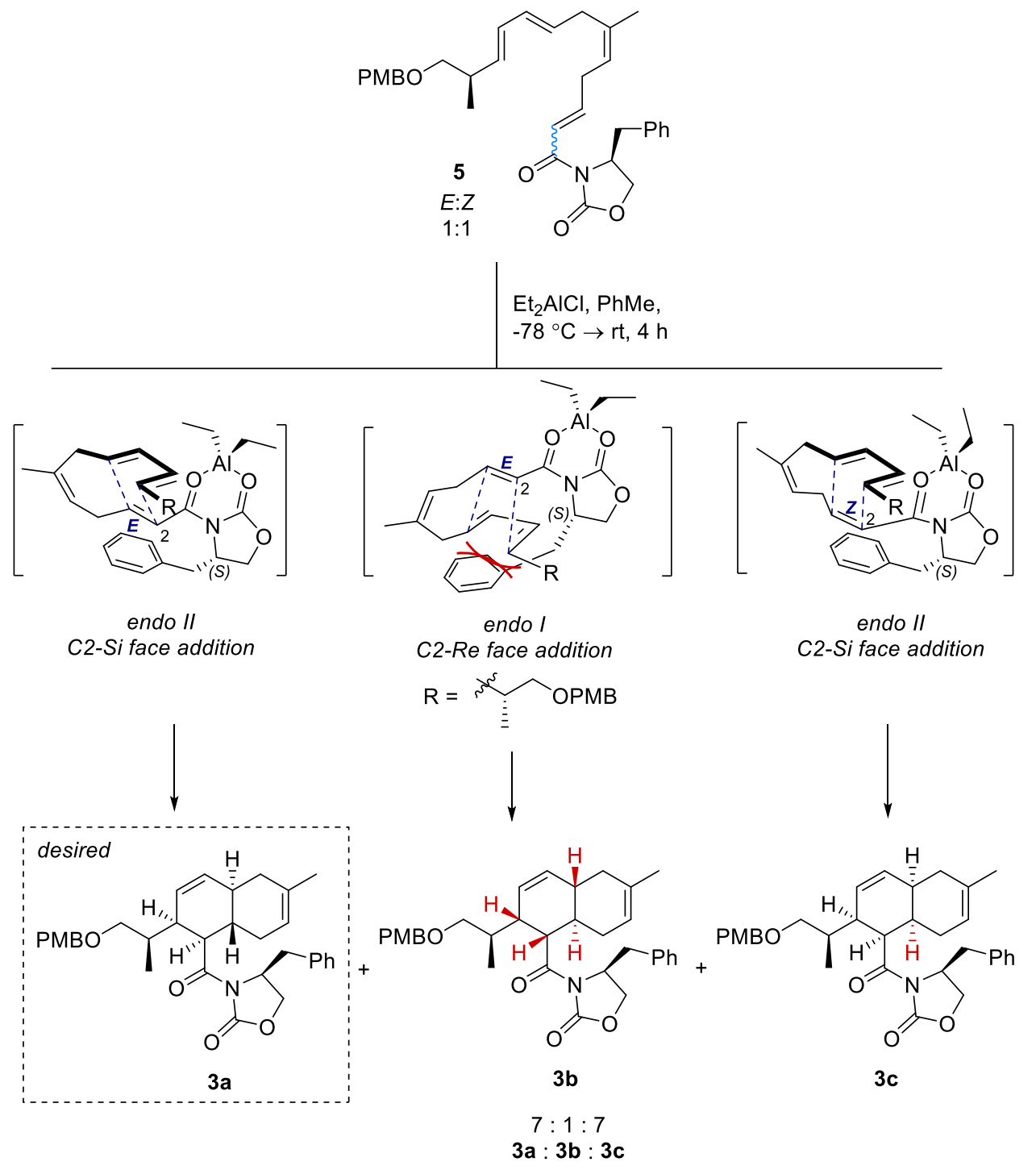

Adducts 3a-c were prepared from tetraene 5 (1.667 $\mathrm{g}, 3.15 \mathrm{mmol})$ according to the procedure described by Brimble and coworkers. ${ }^{1}$ Following purification by flash chromatography on silica gel, eluting with petroleum ether - ethyl acetate $(85: 15)$, the diastereomers were partially separated, yielding a mixture of diastereomers $3 \mathbf{a}$ and $\mathbf{3 b}(7: 1 ; 300 \mathrm{mg}, 0.567 \mathrm{mmol}, 18 \%)$ as a yellow amorphous solid, a pure sample of undesired diastereomer 3c $\mathbf{c}^{\text {a }}$ (258 $\left.\mathrm{mg}, 0.487 \mathrm{mmol}, 16 \%\right)$ as a yellow oil, and combined mixed fractions of epimers 3a and 3c (1:1, 454 $\mathrm{mg}, 0.858 \mathrm{mmol}, 27 \%$ ) as a yellow amorphous solid. All fractions containing 3a were combined following characterisation and used in the next step.

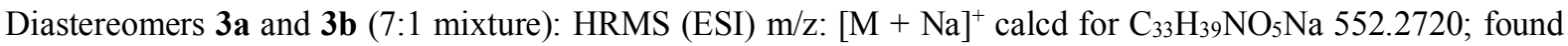
552.2722. Spectroscopic data was concordant with the literature. ${ }^{1}$

Diastereomer 3c $\mathbf{c}^{\mathrm{a}}:[\alpha]_{\mathrm{D}}{ }^{23.0}=+91.7\left(\mathrm{c} 1.32, \mathrm{CHCl}_{3}\right)$; HRMS $(\mathrm{ESI}) \mathrm{m} / \mathrm{z}:[\mathrm{M}+\mathrm{Na}]^{+}$calcd for $\mathrm{C}_{33} \mathrm{H}_{39} \mathrm{NO}_{5} \mathrm{Na}$ 552.2720; found 552.2734; $\mathrm{V}_{\max } / \mathrm{cm}^{-1}$ (neat): 2961, 2852, 1771, 1698, 1513, 1379, 1348, 1245, 1205, 1191, $1085,1033,1014,820,732 ; \delta_{\mathrm{H}}\left(400 \mathrm{MHz}, \mathrm{CDCl}_{3}\right) 7.31-7.24(5 \mathrm{H}, \mathrm{m}, 5 \times \mathrm{ArH}), 7.16-7.13(2 \mathrm{H}, \mathrm{m}, 2 \times \mathrm{ArH})$, 
$6.87(2 \mathrm{H}, \mathrm{d}, J$ 8.6, $2 \times \mathrm{ArH}), 5.91-5.84(2 \mathrm{H}, \mathrm{m}, 2 \times \mathrm{CH}), 5.31(1 \mathrm{H}, \mathrm{brs}, \mathrm{CH}), 4.45-4.40(1 \mathrm{H}, \mathrm{m}, \mathrm{CH}), 4.46$, $4.35\left(2 \mathrm{H}, \mathrm{ABq}, J 11.4, \mathrm{OCH}_{2} \mathrm{Ar}\right), 4.24-4.18(1 \mathrm{H}, \mathrm{m}, \mathrm{CH}), 4.03-3.95\left(2 \mathrm{H}, \mathrm{m}, \mathrm{CH}_{2}\right), 3.80\left(3 \mathrm{H}, \mathrm{s}, \mathrm{OCH}_{3}\right), 3.41$ $\left(1 \mathrm{H}, \mathrm{dd}, J 9.3,4.1,1 / 2 \times \mathrm{CH}_{2}\right), 3.25\left(1 \mathrm{H}, \mathrm{dd}, J 9.3,5.4,1 / 2 \times \mathrm{CH}_{2}\right), 3.18\left(1 \mathrm{H}, \mathrm{dd}, J 13.2,3.0,1 / 2 \times \mathrm{CH}_{2} \mathrm{Ph}\right), 2.50-$ $2.43(2 \mathrm{H}, \mathrm{m}, 2 \times \mathrm{CH}), 2.41-2.40(1 \mathrm{H}, \mathrm{m}, \mathrm{CH}), 2.35\left(2 \mathrm{H}, \mathrm{brs}, \mathrm{CH}_{2}\right), 2.56\left(1 \mathrm{H}, \mathrm{dd}, J 13.2,10.5,1 / 2 \times \mathrm{CH}_{2} \mathrm{Ph}\right)$, $1.98\left(2 \mathrm{H}\right.$, brd, $J$ 6.8, $\left.\mathrm{CH}_{2}\right), 1.92-1.86(1 \mathrm{H}, \mathrm{m}, \mathrm{CH}), 1.56\left(3 \mathrm{H}, \mathrm{s}, \mathrm{CH}_{3}\right), 1.14\left(3 \mathrm{H}, \mathrm{d}, J 6.7, \mathrm{CH}_{3}\right) ; \delta_{\mathrm{C}}(100 \mathrm{MHz}$, $\left.\mathrm{CDCl}_{3}\right) 174.5(\mathrm{CO}), 159.2(\mathrm{C}), 153.7(\mathrm{C}), 136.1(\mathrm{C}), 133.3(\mathrm{C}), 131.2(\mathrm{CH}), 130.9(\mathrm{C}), 129.7(2 \times \mathrm{CH}), 129.5(2$ $\times \mathrm{CH}), 129.0(2 \times \mathrm{CH}), 127.2(\mathrm{CH}), 126.8(\mathrm{CH}), 119.6(\mathrm{CH}), 113.8(2 \times \mathrm{CH}), 73.8\left(\mathrm{CH}_{2}\right), 73.0\left(\mathrm{CH}_{2}\right), 65.8$ $\left(\mathrm{CH}_{2}\right), 57.2(\mathrm{CH}), 55.3\left(\mathrm{OCH}_{3}\right), 43.6(\mathrm{CH}), 40.7(\mathrm{CH}), 37.8\left(\mathrm{CH}_{2} \mathrm{Ph}\right), 36.2(\mathrm{CH}), 34.3(\mathrm{CH}), 32.6\left(\mathrm{CH}_{2}\right), 32.1$ $(\mathrm{CH}), 28.4\left(\mathrm{CH}_{2}\right), 23.7\left(\mathrm{CH}_{3}\right), 16.8\left(\mathrm{CH}_{3}\right)$.

${ }^{a}$ The absolute configuration of $\mathbf{3 c}$ could not be determined unequivocally by 2D NMR spectroscopy due to unresolved bridgehead protons. However, the configuration of $\mathbf{3 c}$ was postulated based on literature precedence, ${ }^{5}$ and further evidence to support this putative assignment was established following PMB deprotection and isolation of $\mathbf{8 c}$ (vide infra).

Alcohol 8a and epimer $(9 R)-\mathbf{8} \mathbf{c}^{\mathrm{a}}$
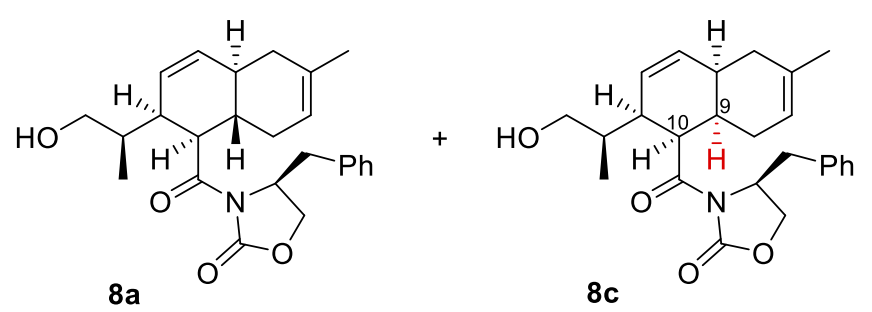

To a stirred mixture of IMDA adducts 3a-c (12:9:1) $(75.0 \mathrm{mg}, 0.142 \mathrm{mmol})$ in dichloromethane $(1.6 \mathrm{~mL})$ and pH7 phosphate buffer $(0.8 \mathrm{~mL})$ was added DDQ $(73 \mathrm{mg}, 0.322 \mathrm{mmol})$ in a single portion. The resulting mixture was stirred vigorously at room temperature for 45 minutes before adding a saturated solution of aq sodium thiosulfate $(10 \mathrm{~mL})$. The layers were separated and the aqueous layer was extracted with ethyl acetate $(3 \times 20$ $\mathrm{mL}$ ). The combined organic extracts were then washed with 1:1 saturated aq sodium bicarbonate/water until the aqueous layer remained colourless $(4 \times 20 \mathrm{~mL})$. The organic layer was then dried using anhydrous $\mathrm{Na}_{2} \mathrm{SO}_{4}$, filtered, and concentrated in vacuo. The crude product was purified by flash chromatography on silica gel, eluting with petroleum ether - ethyl acetate (7:3) to afford alcohol $8 \mathbf{a}(28.9 \mathrm{mg}, 0.0706 \mathrm{mmol}, 50 \%)$ as a yellow amorphous solid and the undesired C9 epimer $8 \mathbf{c}(12.8 \mathrm{mg}, 0.0313 \mathrm{mmol}, 18 \% \mathbf{8 c})$ as a yellow oil (which was contaminated with PMB aldehyde and the suspected minor $\mathbf{8 b}$ diastereomer (approx. $3 \%$ yield)).

8a: $[\alpha]_{\mathrm{D}}{ }^{18}=+246.8\left(\mathrm{c} 0.28, \mathrm{CHCl}_{3}\right.$ ); HRMS (ESI) m/z: $[\mathrm{M}+\mathrm{Na}]^{+}$calcd for $\mathrm{C}_{25} \mathrm{H}_{31} \mathrm{NO}_{4} \mathrm{Na}$ 432.2145; found 432.2146; $\mathrm{v}_{\max } / \mathrm{cm}^{-1}$ (neat): 3527, 2919, 1773, 1678, 1385, 1376, 1347, 1232, 1195, 1098, 1040, 1006, 792, 767, 752,$706 ; \delta_{\mathrm{H}}\left(400 \mathrm{MHz}, \mathrm{CDCl}_{3}\right) 7.36-7.26(5 \mathrm{H}, \mathrm{m}, 5 \times \mathrm{ArH}), 5.71(1 \mathrm{H}, \mathrm{d}, J 10.0, \mathrm{CH}), 5.56(1 \mathrm{H}, \mathrm{ddd}, J 10.0$, 4.6, 2.0, CH), $5.36(1 \mathrm{H}$, brs, $\mathrm{CH}), 4.77-4.71(1 \mathrm{H}, \mathrm{m}, \mathrm{CH}), 4.19-4.11\left(2 \mathrm{H}, \mathrm{m}, \mathrm{CH}_{2} \mathrm{Ph}\right), 3.86(1 \mathrm{H}, \mathrm{dd}, J 11.3$, 6.5, CH), $3.54\left(1 \mathrm{H}\right.$, app.t, $\left.J 9.0,1 / 2 \times \underline{\mathrm{CH}_{2}} \mathrm{OH}\right), 3.48-3.41\left(2 \mathrm{H}, \mathrm{m}, 1 / 2 \times \mathrm{CH}_{2}\right.$ and $\left.1 / 2 \times \underline{\mathrm{CH}_{2}} \mathrm{OH}\right), 3.16-3.12(1 \mathrm{H}$, $\mathrm{m}, \mathrm{CH}), 2.67\left(1 \mathrm{H}, \mathrm{dd}, J 13.2,10.6,1 / 2 \times \mathrm{CH}_{2}\right), 2.32\left(1 \mathrm{H}\right.$, brd, $\left.J 16.5,1 / 2 \times \mathrm{CH}_{2}\right), 2.10-2.02(2 \mathrm{H}, \mathrm{m}, \mathrm{CH}$ and $1 / 2 \times$ $\left.\mathrm{CH}_{2}\right), 1.99(1 \mathrm{H}, \mathrm{dd}, J 11.0,4.4, \mathrm{CH}), 1.85-1.78\left(1 \mathrm{H}, \mathrm{brm}, 1 / 2 \times \mathrm{CH}_{2}\right), 1.71-1.65\left(4 \mathrm{H}, \mathrm{m}, \mathrm{CH}\right.$ and $\left.\mathrm{CH}_{3}\right), 1.57-$ 
$1.50\left(1 \mathrm{H}, \mathrm{m}, 1 / 2 \times \mathrm{CH}_{2}\right), 1.37(1 \mathrm{H}, \mathrm{brs}, \mathrm{OH}), 0.92\left(3 \mathrm{H}, \mathrm{d}, J 6.6, \mathrm{CH}_{3}\right) ; \delta_{\mathrm{C}}\left(100 \mathrm{MHz}, \mathrm{CDCl}_{3}\right) 173.6(\mathrm{C}), 153.2$ (C), $135.7(\mathrm{C}), 134.0(\mathrm{C}), 132.7(\mathrm{CH}), 129.6(2 \times \mathrm{CH}), 129.1(2 \times \mathrm{CH}), 127.4(\mathrm{CH}), 124.3(\mathrm{CH}), 120.9(\mathrm{CH})$, $67.3\left(\mathrm{CH}_{2}\right), 66.2\left(\mathrm{CH}_{2} \mathrm{Ph}\right), 55.6(\mathrm{CH}), 48.4(\mathrm{CH}), 38.4\left(\mathrm{CH}_{2}\right), 37.9(\mathrm{CH}), 37.5(\mathrm{CH}), 37.4\left(\mathrm{CH}_{2}\right), 35.8(\mathrm{CH}), 34.3$ $(\mathrm{CH}), 31.3\left(\mathrm{CH}_{2}\right), 23.5\left(\mathrm{CH}_{3}\right), 14.2\left(\mathrm{CH}_{3}\right)$.

$\mathbf{8} \mathbf{c}^{\mathrm{a}}$ (intractable mixture with suspected minor $\mathbf{8 b}$ diastereomer (7:1) and trace PMB aldehyde): HRMS (ESI) $\mathrm{m} / \mathrm{z}:[\mathrm{M}+\mathrm{Na}]^{+}$calcd for $\mathrm{C}_{25} \mathrm{H}_{31} \mathrm{NO}_{4} \mathrm{Na} 432.2145$; found 432.2155; $\mathrm{V}_{\max } / \mathrm{cm}^{-1}$ (neat): 3529, 2932, 2916, 1770, $1697,1381,1348,1205,1192,1102,1030,910,731 ; \delta_{\mathrm{H}}\left(400 \mathrm{MHz}, \mathrm{CDCl}_{3}\right) 7.36-7.23$ (3H, m, $\left.3 \times \mathrm{ArH}\right), 7.20$ - $7.18(2 \mathrm{H}, \mathrm{m}, 2 \times \mathrm{ArH}), 5.89(2 \mathrm{H}, \mathrm{s}, 2 \times \mathrm{CH}), 5.32-5.31(1 \mathrm{H}, \mathrm{brm}, \mathrm{CH}), 4.44\left(1 \mathrm{H} \text {, app.t, } J 4.7, \mathrm{CH}^{10}\right)^{\mathrm{a}}, 4.28-$ $4.23(1 \mathrm{H}, \mathrm{m}, \mathrm{CH}), 4.17-4.00\left(2 \mathrm{H}, \mathrm{m}, \mathrm{CH}_{2} \mathrm{Ph}\right), 3.62-3.53\left(2 \mathrm{H}, \mathrm{m}, \mathrm{CH}_{2}\right), 3.32\left(1 \mathrm{H}, \mathrm{dd}, J 13.3,3.2,1 / 2 \times \mathrm{CH}_{2}\right)$, $2.64\left(1 \mathrm{H}, \mathrm{dd}, J 13.3,10.3,1 / 2 \times \mathrm{CH}_{2}\right), 2.52-2.41(3 \mathrm{H}, \mathrm{m}, 3 \times \mathrm{CH}), 2.39-2.31\left(2 \mathrm{H}, \mathrm{m}, \mathrm{CH}_{2}\right), 1.99(2 \mathrm{H}, \mathrm{brd}, J$ 8.6, $\left.\mathrm{CH}_{2}\right), 1.87-1.79(1 \mathrm{H}, \mathrm{m}, \mathrm{CH}), 1.58\left(3 \mathrm{H}, \mathrm{s}, \mathrm{CH}_{3}\right), 1.13\left(3 \mathrm{H}, \mathrm{d}, J 6.7, \mathrm{CH}_{3}\right), 1 \times \mathrm{OH}$ not observed; $\delta_{\mathrm{C}}(100$ $\left.\mathrm{MHz}, \mathrm{CDCl}_{3}\right) 174.7$ (C), $154.2(\mathrm{C}), 135.9(\mathrm{C}), 133.4(\mathrm{C}), 131.2(\mathrm{CH}), 129.5(2 \times \mathrm{CH}), 129.0(2 \times \mathrm{CH}), 127.3$ $(\mathrm{CH}), 126.7(\mathrm{CH}), 119.4(\mathrm{CH}), 66.5\left(\mathrm{CH}_{2}\right), 66.0\left(\mathrm{CH}_{2} \mathrm{Ph}\right), 57.5(\mathrm{CH}), 43.2(\mathrm{CH}), 40.7(\mathrm{CH}), 38.0\left(\mathrm{CH}_{2}\right), 36.1$ $(\mathrm{CH}), 36.0(\mathrm{CH}), 32.4\left(\mathrm{CH}_{2}\right), 31.9(\mathrm{CH}), 28.5\left(\mathrm{CH}_{2}\right), 23.7\left(\mathrm{CH}_{3}\right), 16.2\left(\mathrm{CH}_{3}\right)$.

${ }^{\text {a }}$ Careful examination of the ${ }^{1} \mathrm{H}$ NMR spectrum of $\mathbf{8 c}$ showed the $\mathrm{H}^{10}$ resonance exists as an apparent triplet with a coupling constant of $4.7 \mathrm{~Hz}$, indicating a cis relationship between $\mathrm{H}^{10}$ with both $\mathrm{H}^{1}$ and $\mathrm{H}^{9}$, thus supporting the proposed configuration of adducts $\mathbf{3 c}$ and $\mathbf{8 c} .^{6}$ Furthermore, the absolute configuration of $\mathbf{8 c}$ was supported by the NOESY 2D NMR spectrum (see page S27).

$\beta$-Ketoester 9

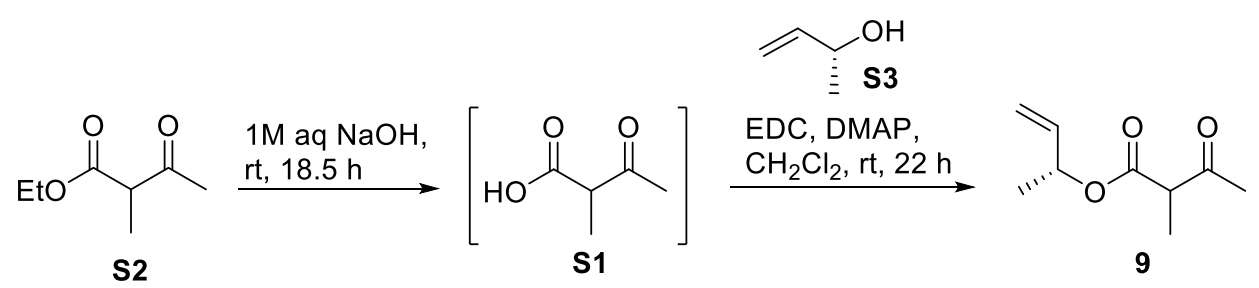

$\alpha$-Methylacetoacetic acid (S1) was prepared with slight variation to the procedure described by Tanaka and coworkers. ${ }^{7}$ Briefly, a solution of ethyl-2-methylacetoacetate (S2) $(0.392 \mathrm{~mL}, 2.77 \mathrm{mmol})$ in $1 \mathrm{M}$ aq sodium hydroxide $(3.33 \mathrm{~mL})$ was stirred vigorously at room temperature for $18.5 \mathrm{~h}$ Upon complete consumption of the starting material, the solution was cooled to $0{ }^{\circ} \mathrm{C}$ and acidified to $\sim \mathrm{pH} 3$ with $1 \mathrm{M}$ aq $\mathrm{HCl}$. The solution was then saturated with sodium chloride and extracted with dichloromethane $(3 \times 50 \mathrm{~mL})$. The organic extracts were dried using anhydrous $\mathrm{Na}_{2} \mathrm{SO}_{4}$, filtered, and concentrated in vacuo at $\sim 10{ }^{\circ} \mathrm{C}$, to afford a crude sample of $\mathbf{S 1}$ (154 mg, $1.33 \mathrm{mmol}, 48 \%$ ) as a colourless oil, which was used immediately due to its reported instability. ${ }^{8} \delta_{\mathrm{H}}$ $\left(400 \mathrm{MHz}, \mathrm{CDCl}_{3}\right) 10.60(1 \mathrm{H}, \mathrm{brs}, \mathrm{OH}), 3.57(1 \mathrm{H}, \mathrm{q}, J 7.3, \mathrm{CH}), 2.30\left(3 \mathrm{H}, \mathrm{s}, \mathrm{CH}_{3}\right), 1.39\left(3 \mathrm{H}, \mathrm{d}, J 7.1, \mathrm{CH}_{3}\right) .{ }^{1} \mathrm{H}$ NMR data is concordant with the literature. ${ }^{7,9}$

To a stirred solution of (R)-3-buten-2-ol (S3) (139 mg, $1.929 \mathrm{mmol})$ and acid $\mathbf{S 1}(154 \mathrm{mg}, 1.33 \mathrm{mmol})$ in dichloromethane $(13 \mathrm{~mL})$ was added 1-ethyl-3-(3-dimethylaminopropyl)carbodiimide hydrochloride salt (305 $\mathrm{mg}, 1.59 \mathrm{mmol})$ and $N, N$-dimethylaminopyridine $(195 \mathrm{mg}, 1.59 \mathrm{mmol})$ and the resulting solution was stirred at 
room temperature for $22 \mathrm{~h}$. Upon complete consumption of the starting material, the solution was diluted with dichloromethane $(50 \mathrm{~mL})$ and washed with a saturated solution of ammonium chloride $(20 \mathrm{~mL})$. The organic layer was then dried using anhydrous $\mathrm{Na}_{2} \mathrm{SO}_{4}$, filtered, and concentrated in vacuo. The crude product was purified by flash chromatography on silica gel, eluting with pentane - ether (4:1) to afford the title compound (107 mg, $0.629 \mathrm{mmol}, 40 \%, 1: 1$ mixture of diastereomers) as a volatile yellow oil. HRMS (ESI) m/z: [M + Na] ${ }^{+}$ calcd for $\mathrm{C}_{9} \mathrm{H}_{14} \mathrm{O}_{3} \mathrm{Na}$ 193.0835; found 193.0837; $\mathrm{V}_{\max } / \mathrm{cm}^{-1}$ (neat): 2987, 2939, 1738, 1714, 1359, 1242, 1200 , 1155, 1043, 991 919, 873; $\delta_{\mathrm{H}}\left(400 \mathrm{MHz}, \mathrm{CDCl}_{3}\right) 5.87-5.78(1 \mathrm{H}, \mathrm{m}, \mathrm{CH}), 5.39(1 \mathrm{H}$, pent, $J$ 6.4, CH), $5.28-$ $5.23\left(1 \mathrm{H}, \mathrm{m}, 1 / 2 \times \mathrm{CH}_{2}\right), 5.18-5.14\left(1 \mathrm{H}, \mathrm{m}, 1 / 2 \times \mathrm{CH}_{2}\right), 3.49(1 \mathrm{H}, \mathrm{q}, J 7.0, \mathrm{CH}), 2.23$ and $2.22\left(3 \mathrm{H}, 2 \times \mathrm{s}, \mathrm{CH}_{3}\right)^{*}$, $1.35-1.32\left(6 \mathrm{H}, \mathrm{m}, 2 \times \mathrm{CH}_{3}\right) ; \delta_{\mathrm{C}}\left(100 \mathrm{MHz}, \mathrm{CDCl}_{3}\right) 203.65$ and 203.63* (C), $169.9(\mathrm{C}), 137.2$ and 137.1* (CH), 116.7 and 116.6* $\left(\mathrm{CH}_{2}\right), 72.3(\mathrm{CH}), 54.01$ and 53.97* $(\mathrm{CH}), 28.54$ and $28.53^{*}\left(\mathrm{CH}_{3}\right), 19.94$ and $19.85^{*}\left(\mathrm{CH}_{3}\right)$, $12.8\left(\mathrm{CH}_{3}\right)$. Spectroscopic data is concordant with the literature. ${ }^{10}$

*1:1 mixture of two diastereomers.

Aldehyde 16

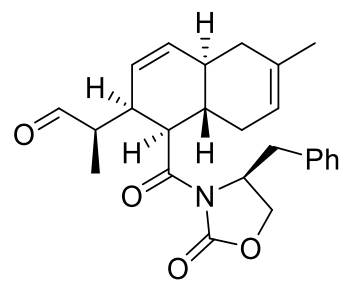

To a stirred solution of alcohol 8a $(40 \mathrm{mg}, 0.0977 \mathrm{mmol})$ in dichloromethane $(3.5 \mathrm{~mL})$ was added sodium bicarbonate $(65.9 \mathrm{mg}, 0.784 \mathrm{mmol})$ followed by Dess-Martin periodinane (DMP) $(83.7 \mathrm{mg}, 0.197 \mathrm{mmol})$ and the resulting mixture was stirred at room temperature for $1 \mathrm{~h}$. Upon complete consumption of the starting material, saturated solutions of sodium bicarbonate $(10 \mathrm{~mL})$ and sodium thiosulfate $(10 \mathrm{~mL})$ were added and the mixture was stirred vigorously for 10 minutes. The layers were then separated and the aqueous layer was extracted with dichloromethane $(3 \times 10 \mathrm{~mL})$. The combined organic extracts were washed with brine $(10 \mathrm{~mL})$, dried using anhydrous $\mathrm{Na}_{2} \mathrm{SO}_{4}$, filtered, and concentrated in vacuo. The crude product was purified by flash chromatography on silica gel, eluting with petroleum ether - ethyl acetate (4:1) to afford the title compound (35.9 mg, $0.0882 \mathrm{mmol}, 90 \%$ ) as a foamy colourless solid. $[\alpha]_{\mathrm{D}}{ }^{19}=+253.8\left(\mathrm{c} 0.16, \mathrm{CHCl}_{3}\right.$ ); HRMS (ESI) m/z: $[\mathrm{M}+\mathrm{Na}]^{+}$calcd for $\mathrm{C}_{25} \mathrm{H}_{29} \mathrm{NO}_{4} \mathrm{Na} 430.1989$; found 430.1988; $\mathrm{v}_{\max } / \mathrm{cm}^{-1}$ (neat): 2924, 1776, 1721, 1689, 1382, 1351, 1211, 1188, 1099, 765, 734; $\delta_{\mathrm{H}}\left(400 \mathrm{MHz}, \mathrm{CDCl}_{3}\right) 9.63(1 \mathrm{H}, \mathrm{s}, \mathrm{CHO}), 7.35-7.31(2 \mathrm{H}, \mathrm{m}, 2 \times \mathrm{ArH}), 7.28$ $-7.23(3 \mathrm{H}, \mathrm{m}, 3 \times \mathrm{ArH}), 5.71(1 \mathrm{H}, \mathrm{d}, J 10.0, \mathrm{CH}), 5.46(1 \mathrm{H}, \mathrm{ddd}, J 10.0,5.0,2.2, \mathrm{CH}), 5.34(1 \mathrm{H}, \mathrm{brs}, \mathrm{CH}), 4.68$ - $4.62(1 \mathrm{H}, \mathrm{m}, \mathrm{CH}), 4.18-4.13\left(2 \mathrm{H}, \mathrm{m}, \mathrm{CH}_{2} \mathrm{Ph}\right), 3.94(1 \mathrm{H}, \mathrm{dd}, J 11.5,6.0, \mathrm{CH}), 3.51-3.46(1 \mathrm{H}, \mathrm{m}, \mathrm{CH}), 3.35$ $\left(1 \mathrm{H}, \mathrm{dd}, J 13.3,3.5,1 / 2 \times \mathrm{CH}_{2}\right), 2.65\left(1 \mathrm{H}, \mathrm{dd}, J 13.3,10.7,1 / 2 \times \mathrm{CH}_{2}\right), 2.54(1 \mathrm{H}, \mathrm{dq}, J 7.2,5.7, \mathrm{CH}), 2.20(1 \mathrm{H}$, brd, $\left.J 16.6,1 / 2 \times \mathrm{CH}_{2}\right), 2.11-2.02\left(2 \mathrm{H}, \mathrm{m}, \mathrm{CH}\right.$ and $\left.1 \frac{1}{2} \times \mathrm{CH}_{2}\right), 1.91-1.76\left(2 \mathrm{H}, \mathrm{m}, \mathrm{CH}\right.$ and $\left.1 / 2 \times \mathrm{CH}_{2}\right), 1.67(3 \mathrm{H}$, s, $\left.\mathrm{CH}_{3}\right), 1.62-1.56\left(1 \mathrm{H}, \mathrm{m}, 1 / 2 \times \mathrm{CH}_{2}\right), 1.14\left(3 \mathrm{H}, \mathrm{d}, J 7.4, \mathrm{CH}_{3}\right) ; \delta_{\mathrm{C}}\left(100 \mathrm{MHz}, \mathrm{CDCl}_{3}\right) 203.7(\mathrm{CHO}), 174.3(\mathrm{C})$, $153.2(\mathrm{C}), 135.8(\mathrm{C}), 134.1(\mathrm{C}), 133.1(\mathrm{CH}), 129.5(2 \times \mathrm{CH}), 129.1(2 \times \mathrm{CH}), 127.4(\mathrm{CH}), 125.3(\mathrm{CH}), 120.6$ $(\mathrm{CH}), 66.3\left(\mathrm{CH}_{2} \mathrm{Ph}\right), 55.7(\mathrm{CH}), 48.1(\mathrm{CH}), 47.1(\mathrm{CH}), 38.1\left(\mathrm{CH}_{2}\right), 37.6(\mathrm{CH}), 37.2\left(\mathrm{CH}_{2}\right), 34.46(\mathrm{CH}), 34.39$ $(\mathrm{CH}), 30.6\left(\mathrm{CH}_{2}\right), 23.5\left(\mathrm{CH}_{3}\right), 11.6\left(\mathrm{CH}_{3}\right)$. 
Vinyl iodide 18<smiles>CC1=CCC(C23CC=C(C)CC2C=CC(C(C)/C=C\I)C3C(=O)N2CCOC2=O)C(CCc2ccccc2)C1</smiles>

To a stirred solution of $\left[\mathrm{Ph}_{3} \mathrm{PCH}_{2} \mathrm{I}\right] \mathrm{I}(\mathbf{1 7})^{11}(774 \mathrm{mg}, 1.46 \mathrm{mmol})$ in THF $(11.5 \mathrm{~mL})$ at $0{ }^{\circ} \mathrm{C}$ was added NaHMDS $(1.0 \mathrm{M}, 1.34 \mathrm{~mL}, 1.34 \mathrm{mmol})$ and the resulting bright orange solution was stirred for 15 minutes. The reaction mixture was then cooled to $-78{ }^{\circ} \mathrm{C}$ before adding aldehyde $16(238 \mathrm{mg}, 0.585 \mathrm{mmol})$ dropwise as a solution in THF $(11.5 \mathrm{~mL})$, and stirring was continued at $-78^{\circ} \mathrm{C}$ for $1.5 \mathrm{~h}$, before being quenched with a saturated solution of ammonium chloride $(30 \mathrm{~mL})$. The mixture was warmed to room temperature, then was extracted with ethyl acetate $(3 \times 50 \mathrm{~mL})$. The combined organic extracts were dried using anhydrous $\mathrm{Na}_{2} \mathrm{SO}_{4}$, filtered, and concentrated in vacuo. The crude product was purified by flash chromatography on silica gel, eluting with petroleum ether - ethyl acetate (9:1) to afford the title compound (197 mg, $0.371 \mathrm{mmol}, 63 \%$ ) as a colourless foam. $[\alpha]_{\mathrm{D}}{ }^{19.9}=+176.9\left(\mathrm{c} 0.13, \mathrm{CHCl}_{3}\right.$ ); HRMS (ESI) m/z: $[\mathrm{M}+\mathrm{Na}]^{+}$calcd for $\mathrm{C}_{26} \mathrm{H}_{30} \mathrm{INO}_{3} \mathrm{Na}$ 554.1163; found $554.1158 ; \mathrm{v}_{\max } / \mathrm{cm}^{-1}$ (neat): $2925,1776,1695,1373,1351,1229,1211,1186,1098,765,731 ; \delta_{\mathrm{H}}(400 \mathrm{MHz}$, $\left.\mathrm{CDCl}_{3}\right) 7.36-7.27(5 \mathrm{H}, \mathrm{m}, 5 \times \mathrm{ArH}), 6.22-6.15(2 \mathrm{H}, \mathrm{m}, 2 \times \mathrm{CH}), 7.74(1 \mathrm{H}, \mathrm{d}, J 10.2, \mathrm{CH}), 5.56(1 \mathrm{H}$, app. ddd, $J$ 10.0, 4.3, 2.2, CH), $5.36-5.35(1 \mathrm{H}, \mathrm{brm}, \mathrm{CH}), 4.77-4.71(1 \mathrm{H}, \mathrm{m}, \mathrm{CH}), 4.17-4.15\left(2 \mathrm{H}, \mathrm{m}, \mathrm{CH}_{2} \mathrm{Ph}\right)$, $3.84(1 \mathrm{H}, \mathrm{dd}, J 11.1,6.9, \mathrm{CH}), 3.73\left(1 \mathrm{H}, \mathrm{dd}, J 13.1,3.4,1 / 2 \times \mathrm{CH}_{2}\right), 3.13-3.09(1 \mathrm{H}, \mathrm{m}, \mathrm{CH}), 2.86(1 \mathrm{H}, \mathrm{dd}, J$ $\left.13.1,11.3,1 / 2 \times \mathrm{CH}_{2}\right), 2.54-2.47(1 \mathrm{H}, \mathrm{m}, \mathrm{CH}), 2.32\left(1 \mathrm{H}\right.$, brd, $\left.J 16.2,1 / 2 \times \mathrm{CH}_{2}\right), 2.07-1.99(3 \mathrm{H}, \mathrm{m}, 2 \times \mathrm{CH}$ and $\left.1 / 2 \times \mathrm{CH}_{2}\right), 1.86-1.79\left(1 \mathrm{H}, \mathrm{m}, 1 / 2 \times \mathrm{CH}_{2}\right), 1.68\left(3 \mathrm{H}, \mathrm{s}, \mathrm{CH}_{3}\right), 1.56-1.49\left(1 \mathrm{H}, \mathrm{m}, 1 / 2 \times \mathrm{CH}_{2}\right), 1.10(3 \mathrm{H}, \mathrm{d}, J 6.8$, $\left.\mathrm{CH}_{3}\right) ; \delta \mathrm{c}\left(100 \mathrm{MHz}, \mathrm{CDCl}_{3}\right) 173.1(\mathrm{CO}), 153.1(\mathrm{CO}), 145.6(\mathrm{CH}), 135.9(\mathrm{C}), 133.8(\mathrm{C}), 133.4(\mathrm{CH}), 129.4(2 \times$ $\mathrm{CH}), 129.0(2 \times \mathrm{CH}), 127.2(\mathrm{CH}), 124.4(\mathrm{CH}), 120.7(\mathrm{CH}), 80.9(\mathrm{CH}), 66.3\left(\mathrm{CH}_{2} \mathrm{Ph}\right), 55.9(\mathrm{CH}), 48.2(\mathrm{CH})$, $41.2(\mathrm{CH}), 39.0\left(\mathrm{CH}_{2}\right), 38.7(\mathrm{CH}), 37.3\left(\mathrm{CH}_{2}\right), 37.1(\mathrm{CH}), 34.3(\mathrm{CH}), 31.2\left(\mathrm{CH}_{2}\right), 23.3\left(\mathrm{CH}_{3}\right), 15.9\left(\mathrm{CH}_{3}\right)$.

\section{Thioester 19}<smiles>CCC(=O)[C@H]1[C@@H](C(C)/C=C\I)C=C[C@H]2CC(C)=CCC12C</smiles>

To a stirred solution of vinyl iodide $18(197 \mathrm{mg}, 0.372 \mathrm{mmol})$ in THF $(25 \mathrm{~mL})$ at $0{ }^{\circ} \mathrm{C}$ was added ethanethiol $(0.20 \mathrm{~mL}, 2.78 \mathrm{mmol})$ followed by ${ }^{n} \mathrm{BuLi}(2 \mathrm{M}$ in cyclohexane, $0.355 \mathrm{~mL}, 0.71 \mathrm{mmol})$, and the resulting solution was stirred at $0{ }^{\circ} \mathrm{C}$ for 30 minutes, then was warmed to room temperature for $3 \mathrm{~h}$. Upon complete consumption of the starting material, the reaction mixture was quenched with water $(10 \mathrm{~mL})$ and extracted with ether $(3 \times 30$ $\mathrm{mL}$ ). The combined organic extracts were dried using anhydrous $\mathrm{Na}_{2} \mathrm{SO}_{4}$, filtered, and concentrated in vacuo. The crude product was purified by flash chromatography on silica gel, eluting with petroleum ether, then petroleum ether - ethyl acetate (95:5) to afford the title compound (118 $\mathrm{mg}, 0.284 \mathrm{mmol}, 76 \%$ ) as a pale yellow oil. $[\alpha]_{\mathrm{D}}^{22.4}=+195.3\left(\mathrm{c} 0.19, \mathrm{CHCl}_{3}\right)$; HRMS (ESI) m/z: $[\mathrm{M}+\mathrm{Na}]^{+}$calcd for $\mathrm{C}_{18} \mathrm{H}_{25} \mathrm{IOSNa}$ 439.0563; found 
439.0556; $\mathrm{v}_{\max } / \mathrm{cm}^{-1}$ (neat): 2963, 2927, 2873, 2830, 1687, 1449, 1375, 1271, 1021, 953, 862, 816, 789, 739; $\delta_{\mathrm{H}}$ $\left(400 \mathrm{MHz}, \mathrm{CDCl}_{3}\right) 6.15-6.11(2 \mathrm{H}, \mathrm{m}, 2 \times \mathrm{CH}) 5.72(1 \mathrm{H}, \mathrm{d}, J 10.1, \mathrm{CH}), 5.54(1 \mathrm{H}$, app. ddd, $J$ 10.0, 4.3, 2.2, $\mathrm{CH}), 5.36-5.34(1 \mathrm{H}, \mathrm{m}, \mathrm{CH}), 2.94\left(2 \mathrm{H}, \mathrm{qd}, J 7.5,1.7, \mathrm{SCH}_{2}\right), 2.86(1 \mathrm{H}, \mathrm{dd}, J 11.4,6.6, \mathrm{CH}), 2.75-2.71(1 \mathrm{H}$, $\mathrm{m}, \mathrm{CH}), 2.62-2.57(1 \mathrm{H}, \mathrm{m}, \mathrm{CH}), 2.26\left(1 \mathrm{H}\right.$, brd, $\left.J 18.0, \frac{1}{2} \times \mathrm{CH}_{2}\right), 2.06-1.96\left(2 \mathrm{H}, \mathrm{m}, 1 / 2 \times \mathrm{CH}_{2}\right.$ and $\left.\mathrm{CH}\right), 1.91$ $(1 \mathrm{H}, \mathrm{qd}, J 10.6,4.5, \mathrm{CH}), 1.80\left(1 \mathrm{H}, \mathrm{brd}, J 14.1,1 / 2 \times \mathrm{CH}_{2}\right), 1.66\left(3 \mathrm{H}, \mathrm{s}, \mathrm{CH}_{3}\right), 1.64-1.58\left(1 \mathrm{H}, \mathrm{m}, 1 / 2 \times \mathrm{CH}_{2}\right)$, $1.32\left(3 \mathrm{H}, \mathrm{t}, J\right.$ 7.5, $\left.\mathrm{SCH}_{2} \underline{\mathrm{CH}_{3}}\right), 1.01\left(3 \mathrm{H}, \mathrm{d}, J 6.6, \mathrm{CH}_{3}\right) ; \delta_{\mathrm{C}}\left(100 \mathrm{MHz}, \mathrm{CDCl}_{3}\right) 200.4(\mathrm{CO}), 145.6(\mathrm{CH}), 133.8$ (C), $133.6(\mathrm{CH}), 124.9(\mathrm{CH}), 121.1(\mathrm{CH}), 80.8(\mathrm{CH}), 58.6(\mathrm{CH}), 41.7(\mathrm{CH}), 40.8(\mathrm{CH}), 37.6(\mathrm{CH}), 37.3\left(\mathrm{CH}_{2}\right)$, $34.8(\mathrm{CH}), 30.7\left(\mathrm{CH}_{2}\right), 23.7\left(\mathrm{SCH}_{2}\right), 23.5\left(\mathrm{CH}_{3}\right), 16.0\left(\mathrm{CH}_{3}\right), 15.0\left(\mathrm{SCH}_{2} \underline{\mathrm{CH}_{3}}\right)$.

Diene 20

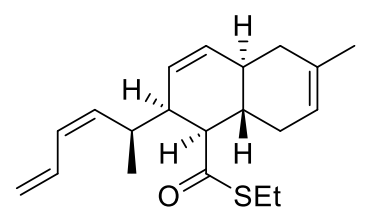

A stirred solution of thioester $19(22.9 \mathrm{mg}, 0.0550 \mathrm{mmol})$ in DMF $(2.2 \mathrm{~mL})$ was degassed with nitrogen by sparging before adding bis(acetonitrile)palladium dichloride $(2.86 \mathrm{mg}, 0.0110 \mathrm{mmol}, 20 \mathrm{~mol} \%)$ followed by tributyl(vinyl)tin $(29 \mu \mathrm{L}, 0.0992 \mathrm{mmol})$ and the resulting solution was stirred at room temperature for $1.5 \mathrm{~h}$. The solution was then diluted with ether $(20 \mathrm{~mL})$ before being washed with $1: 1$ brine/water $(3 \times 10 \mathrm{~mL})$. The organic layer was then dried using anhydrous $\mathrm{Na}_{2} \mathrm{SO}_{4}$, filtered, and concentrated in vacuo. The crude product was purified by flash chromatography on silica gel, eluting with petroleum ether - ethyl acetate (99:1 then 49:1) to afford the title compound (16.6 mg, $0.0525 \mathrm{mmol}, 95 \%)$ as a colourless oil. $[\alpha]_{\mathrm{D}^{24.7}}=+205.6\left(\mathrm{c} 0.09, \mathrm{CHCl}_{3}\right)$; HRMS (ESI) m/z: $[\mathrm{M}+\mathrm{Na}]^{+}$calcd for $\mathrm{C}_{20} \mathrm{H}_{28} \mathrm{OSNa} 339.1753$, found 339.1753; $\mathrm{v}_{\max } / \mathrm{cm}^{-1}$ (neat): 2962, 2927 , $2873,1690,1450,1375,1264,1148,1020,1000,953,903,862,816,789 ; \delta_{\mathrm{H}}\left(500 \mathrm{MHz}, \mathrm{CDCl}_{3}\right) 6.65(1 \mathrm{H}, \mathrm{dtd}$, $J 17.0,10.5,1.2, \mathrm{CH}), 5.89(1 \mathrm{H}, \mathrm{t}, J 11.0, \mathrm{CH}), 5.70(1 \mathrm{H}, \mathrm{d}, J 10.1, \mathrm{CH}), 5.62(1 \mathrm{H}, \mathrm{ddd}, J 10.0,4.1,2.3, \mathrm{CH})$, $5.39(1 \mathrm{H}, \mathrm{t}, J 10.0, \mathrm{CH}), 5.34(1 \mathrm{H}, \mathrm{brs}, \mathrm{CH}), 5.18\left(1 \mathrm{H}, \mathrm{dd}, J 17.2,1.7,1 / 2 \times \mathrm{CH}_{2}\right), 5.08\left(1 \mathrm{H}, \mathrm{d}, J 10.2,1 / 2 \times \mathrm{CH}_{2}\right)$, $3.03-2.96\left(1 \mathrm{H}, \mathrm{m}, 1 / 2 \times \mathrm{SCH}_{2}\right), 2.91-2.84\left(2 \mathrm{H}, \mathrm{m}, \mathrm{CH}\right.$ and $\left.1 / 2 \times \mathrm{SCH}_{2}\right), 2.78-2.74(1 \mathrm{H}, \mathrm{m}, \mathrm{CH}), 2.70-2.67$ $(1 \mathrm{H}, \mathrm{m}, \mathrm{CH}), 2.29\left(1 \mathrm{H}\right.$, brd, $\left.J 15.1,1 / 2 \times \mathrm{CH}_{2}\right), 2.05-1.95\left(2 \mathrm{H}, \mathrm{m}, \mathrm{CH}\right.$ and $\left.1 / 2 \times \mathrm{CH}_{2}\right), 1.90(1 \mathrm{H}$, app.qd, $J$ 10.6, 4.4, CH), $1.78\left(1 \mathrm{H}\right.$, app.brt, $\left.J 13.4,1 / 2 \times \mathrm{CH}_{2}\right), 1.66\left(3 \mathrm{H}, \mathrm{s}, \mathrm{CH}_{3}\right), 1.61-1.56\left(1 \mathrm{H}, \mathrm{m}, 1 / 2 \times \mathrm{CH}_{2}\right), 1.30(3 \mathrm{H}, \mathrm{t}, J$ 7.5, $\left.\mathrm{SCH}_{2} \underline{\mathrm{CH}_{3}}\right), 0.96\left(3 \mathrm{H}, \mathrm{d}, J\right.$ 6.7, $\left.\mathrm{CH}_{3}\right) ; \delta_{\mathrm{C}}\left(125 \mathrm{MHz}, \mathrm{CDCl}_{3}\right) 200.3(\mathrm{CO}), 138.0(\mathrm{CH}), 133.8(\mathrm{C}), 133.0(\mathrm{CH})$, $132.4(\mathrm{CH}), 127.9(\mathrm{CH}), 125.1(\mathrm{CH}), 121.1(\mathrm{CH}), 117.5\left(\mathrm{CH}_{2}\right), 58.9(\mathrm{CH}), 43.7(\mathrm{CH}), 37.6(\mathrm{CH}), 37.3\left(\mathrm{CH}_{2}\right)$, $34.7(\mathrm{CH}), 33.3(\mathrm{CH}), 30.8\left(\mathrm{CH}_{2}\right), 23.5\left(\mathrm{CH}_{3}\right), 23.4\left(\mathrm{SCH}_{2}\right), 17.3\left(\mathrm{CH}_{3}\right), 15.1\left(\mathrm{SCH}_{2} \underline{\mathrm{CH}_{3}}\right)$. 


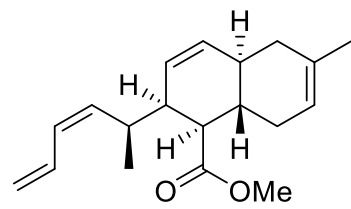

A vial charged with thioester $20(6.5 \mathrm{mg}, 0.0206 \mathrm{mmol})$, silver trifluoroacetoacetate $(22.7 \mathrm{mg}, 0.103 \mathrm{mmol})$ and methanol/dichloromethane $(0.5 \mathrm{~mL}, 4: 1)$ was sealed under nitrogen, and heated to $50{ }^{\circ} \mathrm{C}$ for $16.5 \mathrm{~h}$. The mixture was then diluted with dichloromethane $(5 \mathrm{~mL})$ and washed with a saturated aq solution of sodium bicarbonate (2 $\times 5 \mathrm{~mL}$ ). The organic layer was then dried using anhydrous $\mathrm{Na}_{2} \mathrm{SO}_{4}$, filtered, and concentrated in vacuo. The crude product was purified by flash chromatography on silica gel, eluting with petroleum ether, then petroleum ether - ethyl acetate (97:3) to afford the title compound $(2.0 \mathrm{mg}, 0.00699 \mathrm{mmol}, 34 \%)$ as a yellow oil. $[\alpha]_{\mathrm{D}}^{23.5}=$ +30.0 (c 0.03, $\mathrm{CHCl}_{3}$ ); HRMS (ESI) m/z: [M + Na] $]^{+}$calcd for $\mathrm{C}_{19} \mathrm{H}_{26} \mathrm{O}_{2} \mathrm{Na} 309.1825$; found 309.1822; $\mathrm{Vmax} / \mathrm{cm}^{-1}$ (neat): 2922, 2852, 1740, 1633, 1466, 1378, 1259, 1159, 1093, 1024, 803; $\delta_{\mathrm{H}}\left(500 \mathrm{MHz}, \mathrm{CDCl}_{3}\right) 6.52(1 \mathrm{H}, \mathrm{dtd}$, $J$ 16.7, 10.7, 1.2, CH), $5.90(1 \mathrm{H}, \mathrm{t}, J 11.0, \mathrm{CH}), 5.69-5.67(1 \mathrm{H}, \mathrm{m}, \mathrm{CH}), 5.65-5.62(1 \mathrm{H}, \mathrm{m}, \mathrm{CH}), 5.38-5.34$ $(2 \mathrm{H}, \mathrm{m}, 2 \times \mathrm{CH}), 5.19\left(1 \mathrm{H}, \mathrm{dd}, J 16.4,1.7,1 / 2 \times \mathrm{CH}_{2}\right), 5.11\left(1 \mathrm{H}, \mathrm{d}, J 10.4,1 / 2 \times \mathrm{CH}_{2}\right), 3.68\left(3 \mathrm{H}, \mathrm{s}, \mathrm{OCH}_{3}\right), 2.69-$ $2.63(1 \mathrm{H}, \mathrm{m}, \mathrm{CH}), 2.62-2.60(2 \mathrm{H}, \mathrm{m}, 2 \times \mathrm{CH}), 2.42\left(1 \mathrm{H}\right.$, brd, $\left.J 16.7,1 / 2 \times \mathrm{CH}_{2}\right), 2.05-2.01\left(1 \mathrm{H}, \mathrm{m}, 1 / 2 \times \mathrm{CH}_{2}\right)$, $1.98-1.95(1 \mathrm{H}, \mathrm{m}, \mathrm{CH}), 1.83-1.76\left(2 \mathrm{H}, \mathrm{m}, \mathrm{CH}\right.$ and $\left.1 / 2 \times \mathrm{CH}_{2}\right), 1.67\left(3 \mathrm{H}, \mathrm{s}, \mathrm{CH}_{3}\right), 1.58-1.51(1 \mathrm{H}, \mathrm{m}, 1 / 2 \times$ $\left.\mathrm{CH}_{2}\right), 0.97\left(3 \mathrm{H}, \mathrm{d}, J\right.$ 6.7, $\left.\mathrm{CH}_{3}\right) ; \delta_{\mathrm{C}}\left(125 \mathrm{MHz}, \mathrm{CDCl}_{3}\right) 174.1(\mathrm{CO}), 138.1(\mathrm{CH}), 133.9(\mathrm{C}), 132.7(\mathrm{CH}), 132.2$ $(\mathrm{CH}), 127.9(\mathrm{CH}), 125.6(\mathrm{CH}), 121.1(\mathrm{CH}), 117.7\left(\mathrm{CH}_{2}\right), 51.2\left(\mathrm{OCH}_{3}\right), 49.8(\mathrm{CH}), 42.6(\mathrm{CH}), 37.6(\mathrm{CH}), 37.4$ $\left(\mathrm{CH}_{2}\right), 34.2(\mathrm{CH}), 33.9(\mathrm{CH}), 31.3\left(\mathrm{CH}_{2}\right), 23.5\left(\mathrm{CH}_{3}\right), 18.1\left(\mathrm{CH}_{3}\right)$.

\section{Aldehyde 11}

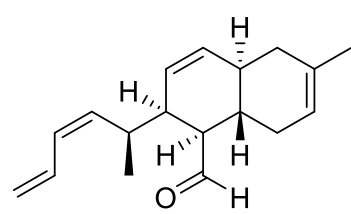

To a stirred solution of thioester $20(24.4 \mathrm{mg}, 0.0772 \mathrm{mmol})$ in dichloromethane $(1 \mathrm{~mL})$ at $-15{ }^{\circ} \mathrm{C}$ was added diisobutylaluminium hydride (1M in cyclohexane, $0.475 \mathrm{~mL}, 0.475 \mathrm{mmol})$ dropwise, and the resulting solution was stirred for 10 minutes before being diluted with dichloromethane $(5 \mathrm{~mL})$ and quenched with a saturated aq solution of sodium bicarbonate $(5 \mathrm{~mL})$. The layers were then separated and the organic layer was dried using anhydrous $\mathrm{Na}_{2} \mathrm{SO}_{4}$, filtered, and concentrated in vacuo to afford the crude alcohol. The crude alcohol was taken up in dichloromethane $(2.8 \mathrm{~mL})$, then sodium bicarbonate $(53.2 \mathrm{mg}, 0.633 \mathrm{mmol})$ and DMP $(67.2 \mathrm{mg}, 0.158$ $\mathrm{mmol}$ ) were added and the mixture was stirred at room temperature for 20 minutes. Saturated solutions of sodium bicarbonate $(10 \mathrm{~mL})$ and sodium thiosulfate $(10 \mathrm{~mL})$ were then added, and the mixture was stirred vigorously for 5 minutes. The layers were then separated and the aqueous layer was extracted with dichloromethane $(2 \times 10 \mathrm{~mL})$. The combined organic extracts were dried using anhydrous $\mathrm{Na}_{2} \mathrm{SO}_{4}$, filtered, and concentrated in vacuo. The crude product was purified by flash chromatography on silica gel, eluting with petroleum ether - ethyl acetate (95:5) to afford the title compound (14.8 $\mathrm{mg}, 0.0578 \mathrm{mmol}, 75 \%$ ) as a yellow oil. 
$[\alpha]_{\mathrm{D}}^{28.4}=+71.1\left(\mathrm{c} 0.045, \mathrm{CHCl}_{3}\right)$; HRMS (ESI) $\mathrm{m} / \mathrm{z}$ : $[\mathrm{M}+\mathrm{Na}]^{+}$calcd for $\mathrm{C}_{18} \mathrm{H}_{24} \mathrm{ONa}$ 279.1719; found 279.1724; $\mathrm{V}_{\max } / \mathrm{cm}^{-1}$ (neat): 2961, 2924, 1717, 1452, 1377, 1074, 999, 904, 790; $\delta_{\mathrm{H}}\left(400 \mathrm{MHz}, \mathrm{CDCl}_{3}\right) 9.75(1 \mathrm{H}$, d, $J$ 4.2, CHO), $6.55(1 \mathrm{H}, \mathrm{dt}, J 16.8,10.6, \mathrm{CH}), 5.92(1 \mathrm{H}, \mathrm{t}, J 11.0, \mathrm{CH}), 5.73(1 \mathrm{H}$, app.ddd, $J$ 10.0, 4.4, 2.0, $\mathrm{CH}), 5.68-5.66(1 \mathrm{H}, \mathrm{m}, \mathrm{CH}), 5.38(1 \mathrm{H}$, brs, $\mathrm{CH}), 5.29(1 \mathrm{H}, \mathrm{t}, J 10.5, \mathrm{CH}), 5.22\left(1 \mathrm{H}, \mathrm{dm}, J 17.0,1 / 2 \times \mathrm{CH}_{2}\right)$, $5.15\left(1 \mathrm{H}, \mathrm{d}, J 10.0,1 / 2 \times \mathrm{CH}_{2}\right), 2.91-2.82(1 \mathrm{H}, \mathrm{m}, \mathrm{CH}), 2.59-2.54(1 \mathrm{H}, \mathrm{m}, \mathrm{CH}), 2.44-2.38(1 \mathrm{H}, \mathrm{m}, \mathrm{CH}), 2.26$ $\left(1 \mathrm{H}\right.$, brd, $\left.J 18.1,1 / 2 \times \mathrm{CH}_{2}\right), 2.06-1.96\left(2 \mathrm{H}, \mathrm{m}, \mathrm{CH}\right.$ and $\left.1 / 2 \times \mathrm{CH}_{2}\right), 1.93(1 \mathrm{H}, \mathrm{dd}, J 11.0,4.4, \mathrm{CH}), 1.85(1 \mathrm{H}$, brd, $J$ 14.7, $\left.1 / 2 \times \mathrm{CH}_{2}\right), 1.68\left(3 \mathrm{H}, \mathrm{s}, \mathrm{CH}_{3}\right), 1.67-1.63\left(1 \mathrm{H}, \mathrm{m}, 1 / 2 \times \mathrm{CH}_{2}\right), 1.02\left(3 \mathrm{H}, \mathrm{d}, J 6.8, \mathrm{CH}_{3}\right) ; \delta_{\mathrm{C}}(100 \mathrm{MHz}$, $\left.\mathrm{CDCl}_{3}\right) 205.9(\mathrm{CHO}), 138.5(\mathrm{CH}), 134.4(\mathrm{C}), 132.4(\mathrm{CH}), 131.9(\mathrm{CH}), 128.8(\mathrm{CH}), 126.9(\mathrm{CH}), 120.7(\mathrm{CH})$, $118.8\left(\mathrm{CH}_{2}\right), 56.2(\mathrm{CH}), 43.7(\mathrm{CH}), 38.1(\mathrm{CH}), 37.0\left(\mathrm{CH}_{2}\right), 34.8(\mathrm{CH}), 32.9(\mathrm{CH}), 30.7\left(\mathrm{CH}_{2}\right), 23.6\left(\mathrm{CH}_{3}\right), 19.1$ $\left(\mathrm{CH}_{3}\right)$.

Tricarbonyl 21

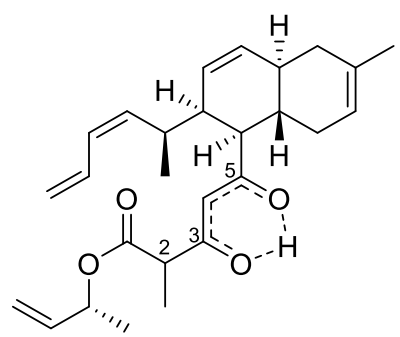

To a stirred solution of sodium hydride ( $60 \%$ dispersion in mineral oil, $4.1 \mathrm{mg}, 0.102 \mathrm{mmol})$ in THF $(0.4 \mathrm{~mL})$ at $-10{ }^{\circ} \mathrm{C}$ under argon was added $\beta$-ketoester $9(17.3 \mathrm{mg}, 0.0 .102 \mathrm{mmol})$ as a solution in THF $(0.4 \mathrm{~mL})$. The solution was stirred for 10 minutes before adding ${ }^{n} \mathrm{BuLi}(1.48 \mathrm{M}$ in cyclohexane, $0.069 \mathrm{~mL}, 0.102 \mathrm{mmol})$, and stirring was continued for a further 10 minutes at $-10{ }^{\circ} \mathrm{C}$. Aldehyde $11(5.1 \mathrm{mg}, 0.0199 \mathrm{mmol})$ was then added dropwise as a solution in THF $(0.2 \mathrm{~mL})$, and the reaction mixture was stirred at $-10^{\circ} \mathrm{C}$ for 20 minutes, before being quenched with water $(0.5 \mathrm{~mL})$. The mixture was then diluted with ether $(5 \mathrm{~mL})$, and acidified to $\sim \mathrm{pH} 4$ with $2 \mathrm{M}$ aq hydrochloric acid. The layers were separated and the aqueous layer was extracted with ether $(3 \times 5$ $\mathrm{mL}$ ). The combined organic extracts were dried using anhydrous $\mathrm{Na}_{2} \mathrm{SO}_{4}$, filtered, and concentrated in vacuo. The crude aldol product was then dissolved in dichloromethane $(1 \mathrm{~mL})$, and sodium bicarbonate $(11.0 \mathrm{mg}, 0.131$ $\mathrm{mmol}$ ) and DMP (13.9 mg, $0.0328 \mathrm{mmol})$ were added. The mixture was then stirred at room temperature for 30 minutes before adding saturated solutions of sodium bicarbonate $(2 \mathrm{~mL})$ and sodium thiosulfate $(2 \mathrm{~mL})$, and the resulting mixture was stirred vigorously for 5 minutes. The layers were then separated and the aqueous layer was extracted with dichloromethane $(2 \times 5 \mathrm{~mL})$. The combined organic extracts were dried using anhydrous $\mathrm{Na}_{2} \mathrm{SO}_{4}$, filtered, and concentrated in vacuo. The crude product was purified by flash chromatography on silica gel, eluting with petroleum ether - ethyl acetate (95:5) to afford the title compound (2.5 $\mathrm{mg}, 0.00589 \mathrm{mmol}$, $30 \%, 1: 1$ mixture of $\mathrm{C}^{2}$ epimers) as a pale yellow oil. HRMS (ESI) $\mathrm{m} / \mathrm{z}$ : $[\mathrm{M}+\mathrm{Na}]^{+}$calcd ${ }^{2} \mathrm{C}_{27} \mathrm{H}_{36} \mathrm{O}_{4} \mathrm{Na}$ 447.2506; found 447.2498; $\mathrm{v}_{\max } / \mathrm{cm}^{-1}$ (neat): 2959, 2925, 1738, 1599, 1456, 1377, 1191, 1083, 1043, 996, 903, 791; $\delta_{\mathrm{H}}\left(500 \mathrm{MHz}, \mathrm{CDCl}_{3}\right) 15.37\left(1 \mathrm{H}, \mathrm{brs}, \mathrm{C}^{3 / 5} \mathrm{OH}\right), 6.48(1 \mathrm{H}, \mathrm{dt}, J 16.8,10.7, \mathrm{CH}), 5.87-5.77(2 \mathrm{H}, \mathrm{m}, 2 \times$ $\mathrm{CH}), 5.71(1 \mathrm{H}$, brd, $J 7.2, \mathrm{CH}), 5.66-5.63(2 \mathrm{H}, \mathrm{m}, 2 \times \mathrm{CH}), 5.41-5.35(3 \mathrm{H}, \mathrm{m}, 3 \times \mathrm{CH}), 5.25(1 \mathrm{H}, \mathrm{ddt}, J 17.3$, 5.9, $\left.1.3,1 / 2 \times \mathrm{CH}=\underline{\mathrm{CH}_{2}}\right), 5.17-5.11\left(2 \mathrm{H}, \mathrm{m}, 2 \times 1 / 2 \times \mathrm{CH}=\underline{\mathrm{CH}_{2}}\right), 5.06\left(1 \mathrm{H}, \operatorname{brd}, J 9.9,1 / 2 \times \mathrm{CH}=\underline{\mathrm{CH}_{2}}\right), 3.39(1 \mathrm{H}$, q, $J 7.1, \mathrm{CH}), 2.79-2.74(1 \mathrm{H}, \mathrm{brm}, \mathrm{CH}), 2.52-2.48(2 \mathrm{H}, \mathrm{m}, 2 \times \mathrm{CH}), 2.34\left(1 \mathrm{H}\right.$, brd, $\left.J 16.9,1 / 2 \times \mathrm{CH}_{2}\right), 2.05-$ 
$1.99\left(2 \mathrm{H}, \mathrm{m}, 1 / 2 \times \mathrm{CH}_{2}\right.$ and $\left.\mathrm{CH}\right), 1.88-1.78\left(2 \mathrm{H}, \mathrm{m}, 1 / 2 \times \mathrm{CH}_{2}\right.$ and $\left.\mathrm{CH}\right), 1.67\left(3 \mathrm{H}, \mathrm{s}, \mathrm{CH}_{3}\right), 1.54(1 \mathrm{H}, \mathrm{m}, 1 / 2 \times$ $\left.\mathrm{CH}_{2}\right)^{\mathrm{a}}, 1.42\left(3 \mathrm{H}, \mathrm{d}, J 6.8, \mathrm{CH}_{3}\right)$ and $1.32\left[6 \mathrm{H}\right.$, app.t, $J$ 6.7, $\mathrm{CH}_{3}$ (two diastereomers)] ${ }^{*}, 0.97\left(3 \mathrm{H}, \mathrm{d}, J 6.7, \mathrm{CH}_{3}\right)$; $\delta_{\mathrm{C}}\left(125 \mathrm{MHz}, \mathrm{CDCl}_{3}\right) 194.2$ and $194.0(\mathrm{C})^{*}, 192.1$ and $191.9(\mathrm{C})^{*}, 170.21$ and $170.17(\mathrm{C})^{*}, 138.2$ and 138.1 $(\mathrm{CH})^{*}, 137.3$ and $137.2(\mathrm{CH})^{*}, 134.0(\mathrm{C}), 132.9$ and $132.8(\mathrm{CH})^{*}, 132.3$ and $132.2(\mathrm{CH})^{*}, 127.8(\mathrm{CH}), 125.6$ and $125.5(\mathrm{CH}), 121.1(\mathrm{CH}), 117.7\left(\mathrm{CH}_{2}\right), 116.4$ and $116.3\left(\mathrm{CH}_{2}\right)^{*}, 100.2$ and $100.0(\mathrm{CH})^{*}, 72.2$ and 72.1 $(\mathrm{CH})^{*}, 52.5$ and $52.4(\mathrm{CH})^{*}, 49.7$ and $49.5(\mathrm{CH})^{*}, 44.5(\mathrm{CH}), 37.8(\mathrm{CH}), 37.4\left(\mathrm{CH}_{2}\right), 33.7$ and $33.6(\mathrm{CH}), 33.4$ and $33.3(\mathrm{CH}), 31.24$ and $31.22\left(\mathrm{CH}_{2}\right)^{*}, 23.5\left(\mathrm{CH}_{3}\right), 20.0$ and $19.9\left(\mathrm{CH}_{3}\right), 17.82$ and $17.79\left(\mathrm{CH}_{3}\right), 14.2$ and 14.1 $\left(\mathrm{CH}_{3}\right)$.

${ }^{\text {a }}$ signal occluded by water peak, assignment made using HSQC and COSY 2D NMR experiments; * the two epimers could not be differentiated due to their 1:1 ratio and close proximity of their peaks in both the ${ }^{1} \mathrm{H}$ and ${ }^{13} \mathrm{C}$ NMR spectra.

Anthracimycin ((-)-1)

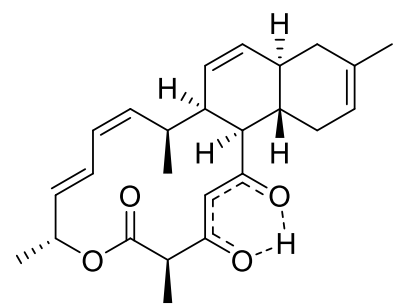

A solution of tricarbonyl $21(2.0 \mathrm{mg}, 0.00471 \mathrm{mmol})$ in dichloromethane $(2.4 \mathrm{~mL})$ was degassed with nitrogen by sparging for 5 minutes, before adding Grubbs I catalyst $(0.76 \mathrm{mg}, 0.000926 \mathrm{mmol}, 20 \mathrm{~mol} \%)$. The solution was then heated to reflux for 1.5 hours, before being cooled to room temperature. 1,8-Diazabicyclo[5.4.0]undec7-ene (DBU), $(0.0144 \mathrm{~mL}, 0.0965 \mathrm{mmol})$ was then added, and the solution was stirred at room temperature for 30 minutes. The reaction was then diluted with dichloromethane $(10 \mathrm{~mL})$, and washed with 1:1 saturated aq ammonium chloride and water $(3 \times 10 \mathrm{~mL})$. The organic layer was then dried using anhydrous $\mathrm{Na}_{2} \mathrm{SO}_{4}$, filtered, and concentrated in vacuo. The crude product was then purified by RP-silica gel 60 C18-preperative TLC $^{\text {a }}$ eluting with acetonitrile - water (97:3) to provide the title compound $(0.77 \mathrm{mg}, 0.00194 \mathrm{mmol}, 37 \%$ yield accounting for trace impurities) as a white solid. For analytical purposes and biological testing, the crude material could also be purified by RP-HPLC (Phenomenex Gemini ${ }^{\circledR}$ C18 column, $5 \mu \mathrm{m}, 110 \AA, 150 \times 4.6 \mathrm{~mm}$ ) using a gradient of $\mathrm{H}_{2} \mathrm{O}: \mathrm{MeCN}$ (1:1 to 0:1 over 60 minutes, $\left.1 \mathrm{~mL} / \mathrm{min}\right)$, with UV detection at 210, 236, 254 and $280 \mathrm{~nm}$. Under these conditions, anthracimycin (1) $\left(0.4 \mathrm{mg}, 0.00101 \mathrm{mmol}, \mathrm{t}_{\mathrm{R}} 37.8 \mathrm{~min}\right)$ was obtained as a white solid. $[\alpha]_{\mathrm{D}^{21.4}}=-235.0\left(\mathrm{c} 0.02, \mathrm{CDCl}_{3}\right)\left[\right.$ lit. $\left.^{12}-333.0\left(\mathrm{c} 0.1, \mathrm{CDCl}_{3}\right)\right]$; HRMS (ESI) m/z: $[\mathrm{M}+\mathrm{Na}]^{+}$calcd for $\mathrm{C}_{25} \mathrm{H}_{32} \mathrm{O}_{4} \mathrm{Na}$ 419.2193; found 419.2186; $\mathrm{V}_{\max } / \mathrm{cm}^{-1}$ (neat): 2920, 2851, 1729, 1633, 1463, 1377, 1261, 1080, 1020, 802; For ${ }^{1} \mathrm{H}$ and ${ }^{13} \mathrm{C}$ NMR data see tables S1 and S2.

${ }^{a}$ Anthracimycin was observed to be partially unstable on silica gel, and as such, required purification under reverse phase conditions. 
Table S2. ${ }^{1} \mathrm{H}$ NMR comparison of natural anthracimycin $((-)-\mathbf{1})^{12}$ with synthetic anthracimycin ((-)1).

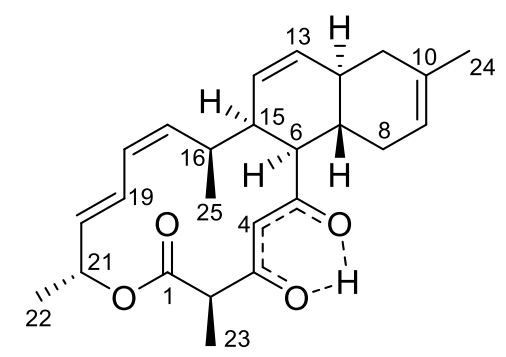

\begin{tabular}{|c|c|c|c|}
\hline $\mathbf{H}$ & $\begin{array}{c}\text { Natural (-)-1 } \\
\delta_{\mathrm{H}}\left(500 \mathrm{MHz}, \mathrm{CDCl}_{3}\right)\end{array}$ & $\begin{array}{c}\text { Synthetic (-)-1 } \\
\delta_{\mathrm{H}}\left(500 \mathrm{MHz}, \mathrm{CDCl}_{3}\right)\end{array}$ & $\Delta \delta$ \\
\hline 2 & $3.53(\mathrm{q}, J 7.0)$ & $3.53(\mathrm{q}, J 7.0)$ & 0 \\
\hline 4 & $5.96(\mathrm{~s})$ & $5.96(\mathrm{~s})$ & 0 \\
\hline 3/5-OH & Not observed & 15.45 (brs) & NA \\
\hline 6 & $2.58(\mathrm{dd}, J 11.8,6.7)$ & $2.66-2.57(\mathrm{~m})^{\mathrm{b}}$ & $-{ }^{b}$ \\
\hline 7 & $1.98(\mathrm{ddd}, J 14.5,12.5,11.8)$ & $2.00-1.97(\mathrm{~m})$ & +0.01 \\
\hline $8 \alpha$ & $2.39(\mathrm{ddd}, J 16.0,4.5,4.5)$ & $2.39(\mathrm{brd}, J 18.6)$ & 0 \\
\hline $8 \beta$ & $1.52(\mathrm{ddd}, J 16.0,10.5,4.5)$ & Not observed $^{\mathrm{c}}$ & NA \\
\hline 9 & $5.36(\mathrm{brd}, J 4.5)$ & 5.36 (brd, $J 4.6)$ & 0 \\
\hline $11 \alpha$ & $2.02(\mathrm{dd}, J 16.5,4.0)$ & $2.03(\mathrm{brd}, J 5.3)$ & +0.01 \\
\hline $11 \beta$ & $1.82(\mathrm{dd}, J 16.5,10.3)$ & $1.85-1.81(\mathrm{~m})$ & +0.01 \\
\hline 12 & $2.64^{\mathrm{a}}$ & $2.66-2.57(\mathrm{~m})^{\mathrm{b}}$ & $-{ }^{b}$ \\
\hline 13 & $5.71(\mathrm{~d}, J 10.0)$ & $5.72(\mathrm{~d}, J 10.4)$ & +0.01 \\
\hline 14 & $5.53(\mathrm{dd}, J 10.0,5.0)$ & $5.54-5.52(\mathrm{~m})$ & 0 \\
\hline 15 & $2.60^{\mathrm{a}}$ & $2.66-2.57(\mathrm{~m})^{\mathrm{b}}$ & $-b$ \\
\hline 16 & $1.93(\mathrm{~m})$ & $1.93(\mathrm{dd}, J 10.6,4.2)$ & 0 \\
\hline 17 & $5.40(\mathrm{dd}, J 10.5,9.8)$ & $5.40($ app. t, $J 9.4)$ & 0 \\
\hline 18 & $5.87(\mathrm{dd}, J 11.0,10.5)$ & 5.87 (app. t, $J 10.9$ ) & 0 \\
\hline 19 & $6.45(\mathrm{dd}, J 15.2,11.0)$ & $6.46(\mathrm{ddd}, J 15.4,11.6,1.8)$ & +0.01 \\
\hline 20 & $5.56(\mathrm{dd}, J 15.2,2.4)$ & $5.58-5.56(\mathrm{~m})^{\mathrm{b}}$ & $-b$ \\
\hline 21 & $5.57(\mathrm{dq}, J 6.5,2.4)$ & $5.58-5.56(\mathrm{~m})^{\mathrm{b}}$ & $-b$ \\
\hline 22 & $1.33(\mathrm{~d}, J 6.5)$ & $1.34(\mathrm{~d}, J 6.8)$ & +0.01 \\
\hline 23 & $1.39(\mathrm{~d}, J 7.0)$ & $1.40(\mathrm{~d}, J 6.8)$ & +0.01 \\
\hline 24 & $1.67(\mathrm{~s})$ & $1.67(\mathrm{~s})$ & 0 \\
\hline 25 & $0.94(\mathrm{~d}, J 7.0)$ & $0.95(\mathrm{~d}, J 6.6)$ & +0.01 \\
\hline
\end{tabular}

${ }^{a}$ The coupling constant was not measured because of overlapping signals

bUnresolved signals

${ }^{\mathrm{c}}$ Not observed due to overlapping water signal 
Table S3. ${ }^{13} \mathrm{C}$ NMR comparison of natural anthracimycin $((-)-1)^{12}$ with synthetic anthracimycin ((-)1).

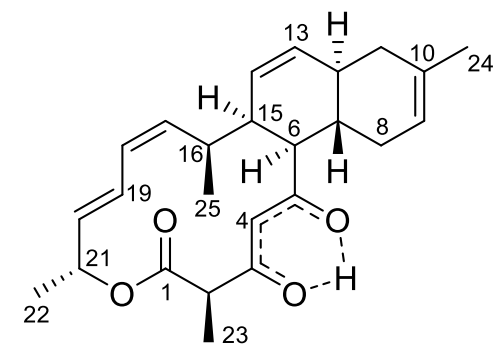

\begin{tabular}{|c|c|c|c|}
\hline C & $\begin{array}{c}\text { Natural (-)-1 } \\
\delta_{\mathrm{C}}\left(125 \mathrm{MHz}, \mathrm{CDCl}_{3}\right)\end{array}$ & $\begin{array}{c}\text { Synthetic (-)-1 } \\
\delta_{\mathrm{C}}\left(125 \mathrm{MHz}, \mathrm{CDCl}_{3}\right)\end{array}$ & $\Delta \delta$ \\
\hline 1 & 168.9 & 168.9 & 0 \\
\hline 2 & 49.2 & 49.2 & 0 \\
\hline 3 & 190.9 & 190.8 & -0.1 \\
\hline 4 & 103.0 & 103.0 & 0 \\
\hline 5 & 194.1 & 194.2 & +0.1 \\
\hline 6 & 52.6 & 52.7 & +0.1 \\
\hline 7 & 37.4 & 37.4 & 0 \\
\hline 8 & 31.4 & 31.4 & 0 \\
\hline 9 & 121.0 & 121.0 & 0 \\
\hline 10 & 134.0 & 134.0 & 0 \\
\hline 11 & 37.5 & 37.5 & 0 \\
\hline 12 & 33.0 & 33.0 & 0 \\
\hline 13 & 133.0 & 133.0 & 0 \\
\hline 14 & 124.9 & 124.9 & 0 \\
\hline 15 & 46.0 & 46.0 & 0 \\
\hline 16 & 32.8 & 32.8 & 0 \\
\hline 17 & 139.1 & 139.1 & 0 \\
\hline 18 & 126.1 & 126.1 & 0 \\
\hline 19 & 123.7 & 123.8 & +0.1 \\
\hline 20 & 131.7 & 131.7 & 0 \\
\hline 21 & 70.0 & 70.0 & 0 \\
\hline 22 & 21.0 & 21.0 & 0 \\
\hline 23 & 11.8 & 11.8 & 0 \\
\hline 24 & 23.5 & 23.5 & 0 \\
\hline 25 & 16.4 & 16.4 & 0 \\
\hline
\end{tabular}




\section{References}

(1) Freeman, J. L.; Brimble, M. A.; Furkert, D. P. Org. Chem. Front. 2019, 6, 2954-2963.

(2) Evans, D. A.; Black, W. C. J. Am. Chem. Soc. 1993, 115, 4497-4513.

(3) Könning, D.; Hiller, W.; Christmann, M. Org. Lett. 2012, 14, 5258-5261.

(4) Shioiri, T.; Noguchi, H.; Aoyama, T. Heterocycles 2002, 58, 471.

(5) Roush, W. R.; Gillis, H. R. J. Org. Chem. 1982, 47, 4825-4829.

(6) Roush, W. R.; Champoux, J. A.; Peterson, B. C. Tetrahedron Lett. 1996, 37, 8989-8992.

(7) Tanaka, M.; Oota, O.; Hiramatsu, H.; Fujiwara, K. Bull. Chem. Soc. Jpn. 1988, 61, 2473-2479.

(8) Bertogg, A.; Hintermann, L.; Huber, D. P.; Perseghini, M.; Sanna, M.; Togni, A. Helv. Chim. Acta 2012, 95, 353-403.

(9) Anwar, M.; Cowley, A. R.; Moloney, M. G. Tetrahedron Asymmetry 2010, 21, 1758-1770.

(10) Gilbert, J. C.; Kelly, T. A. J. Org. Chem. 1988, 53, 449-450.

(11) Dias, L. C.; Ferreira, M. A. B. J. Org. Chem. 2012, 77, 4046-4062.

(12) Jang, K. H.; Nam, S.-J.; Locke, J. B.; Kauffman, C. A.; Beatty, D. S.; Paul, L. A.; Fenical, W. Angew. Chem. Int. Ed. 2013, 52, 7822-7824. 


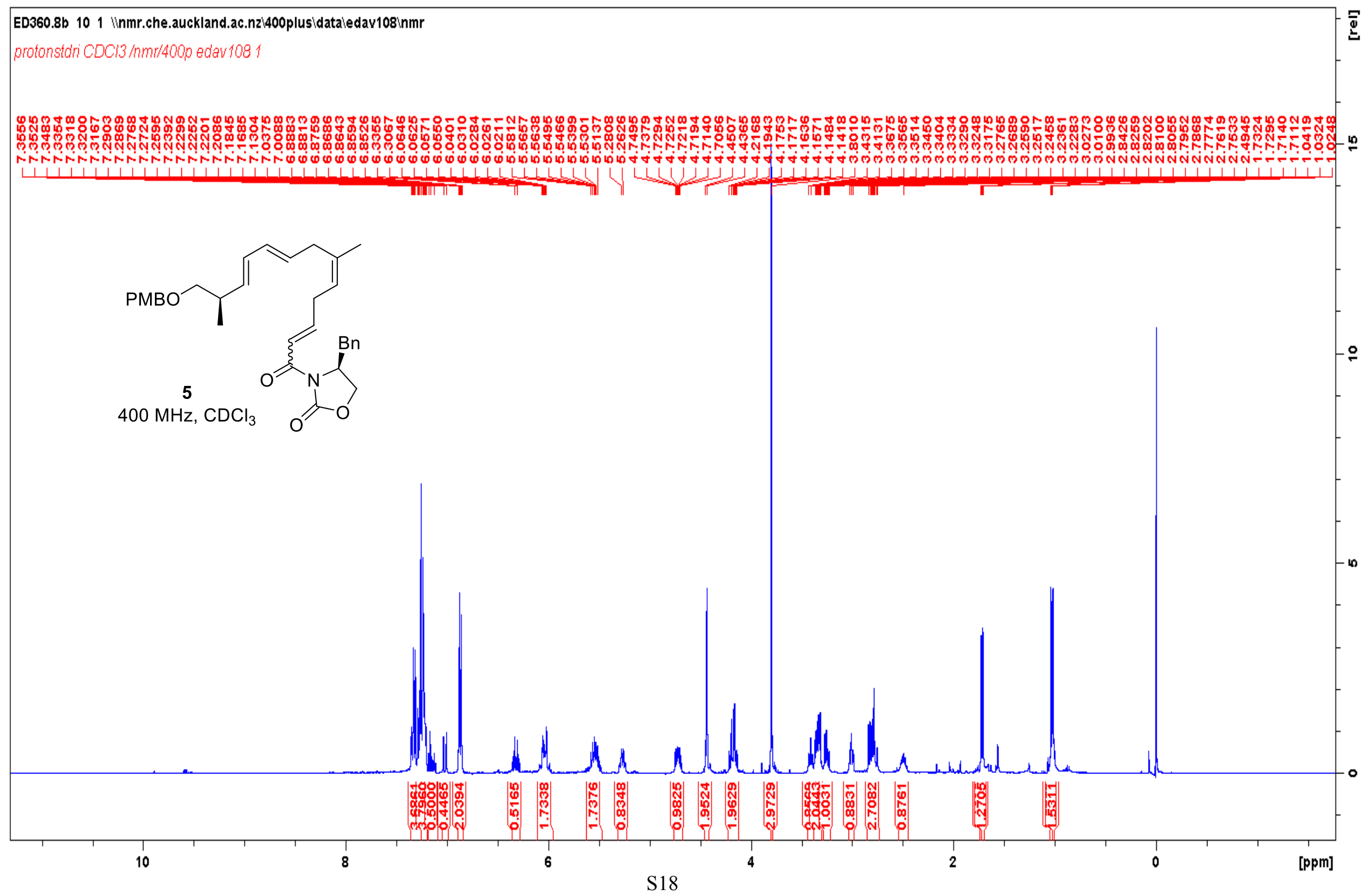




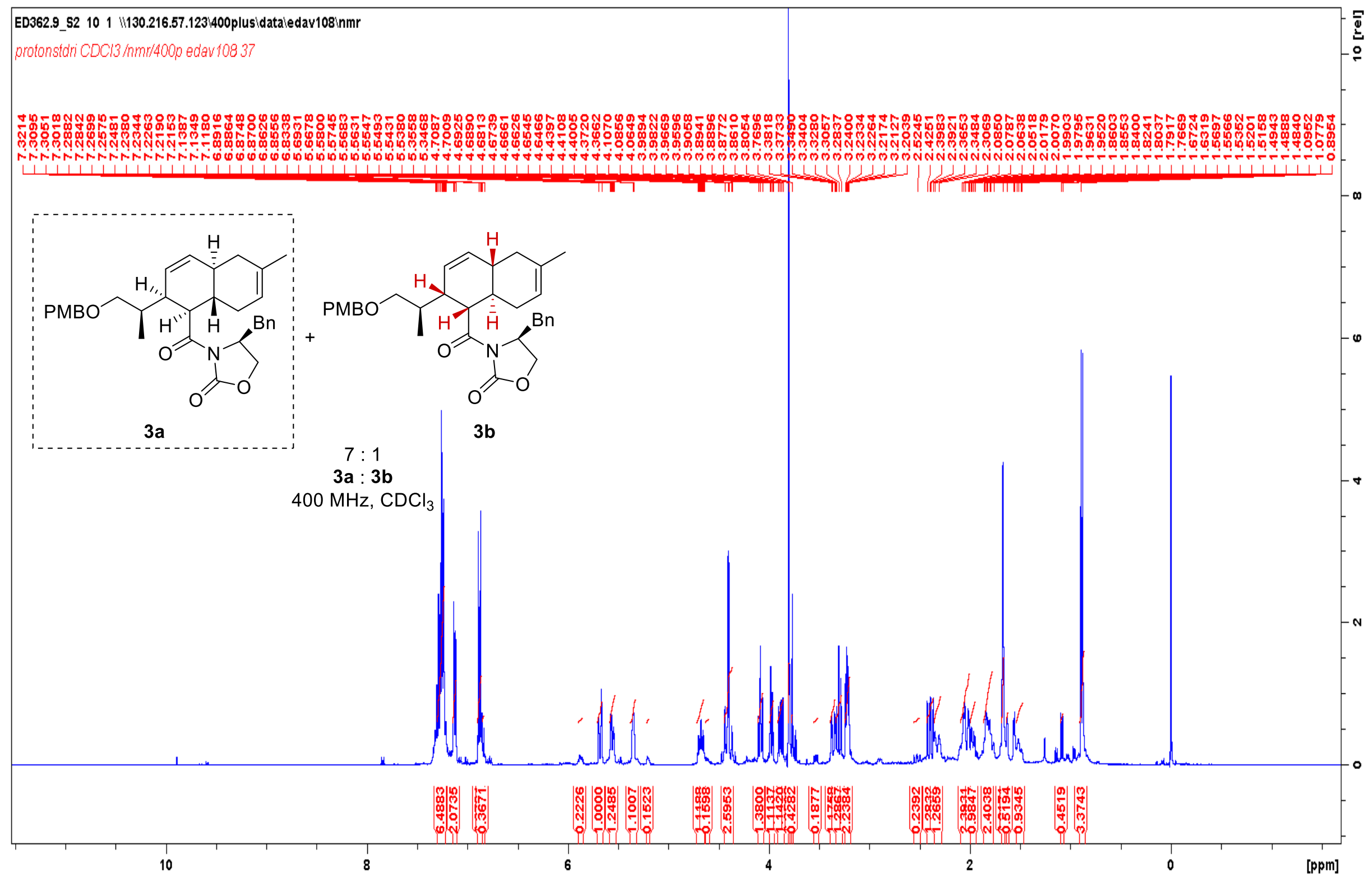




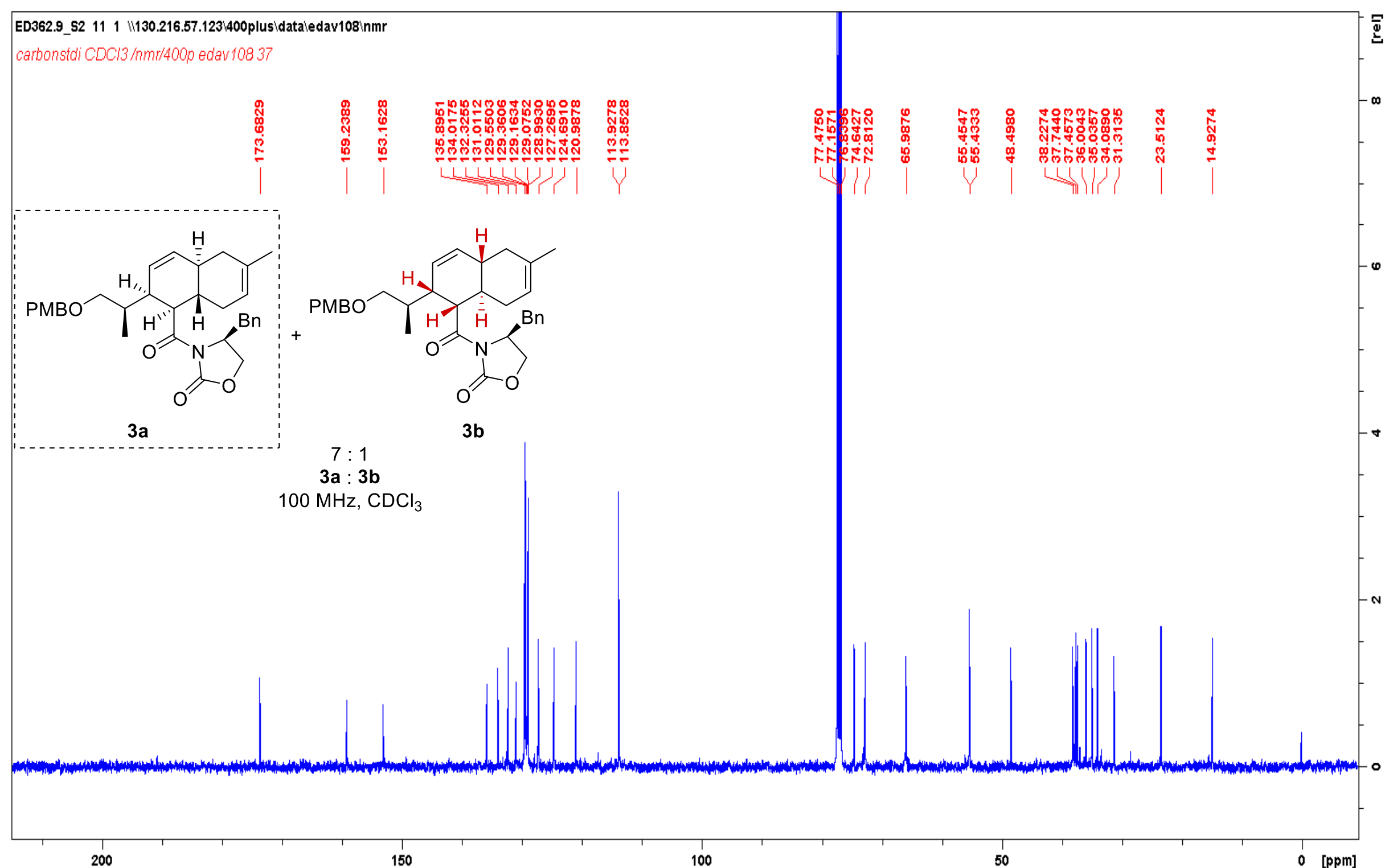




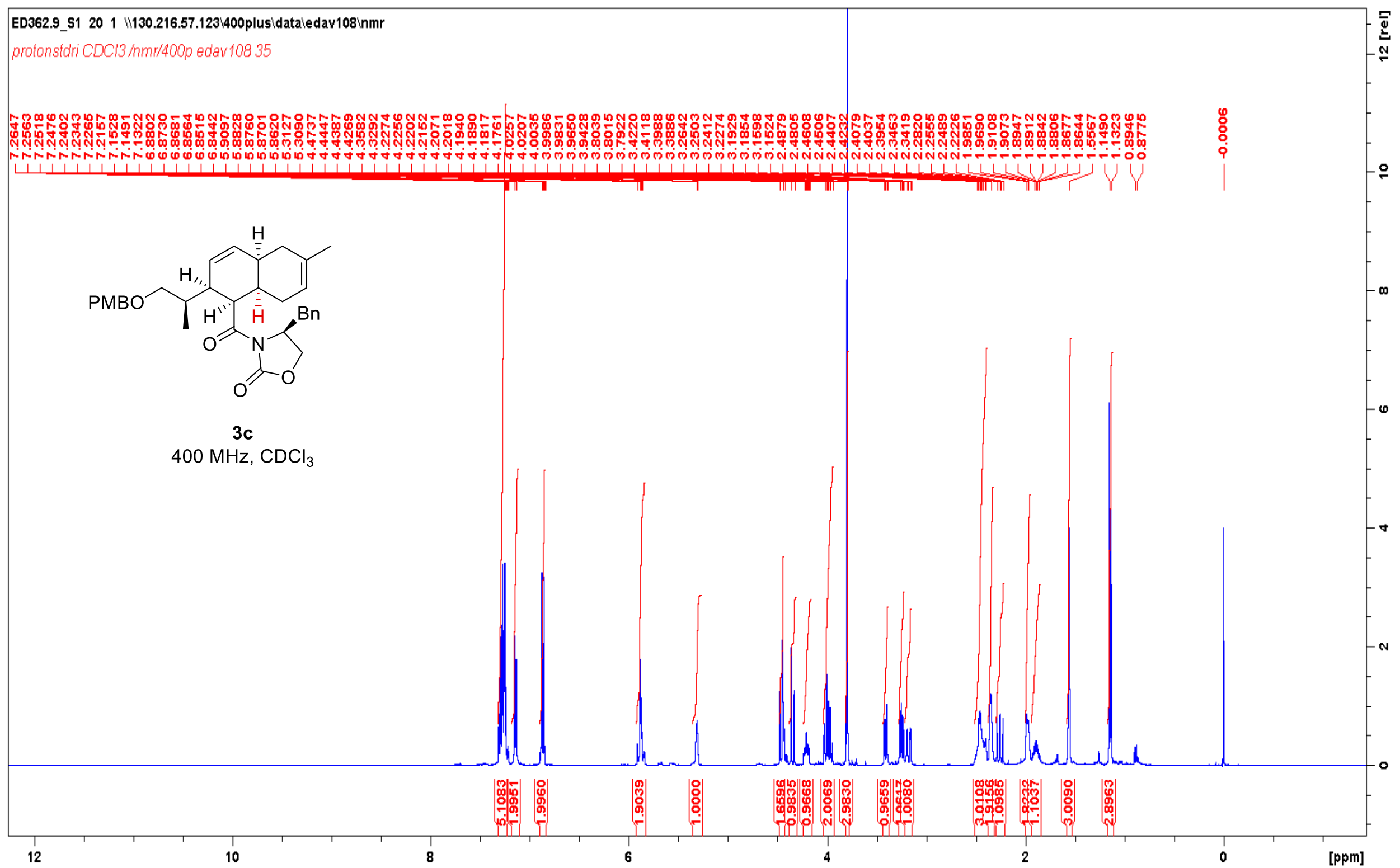




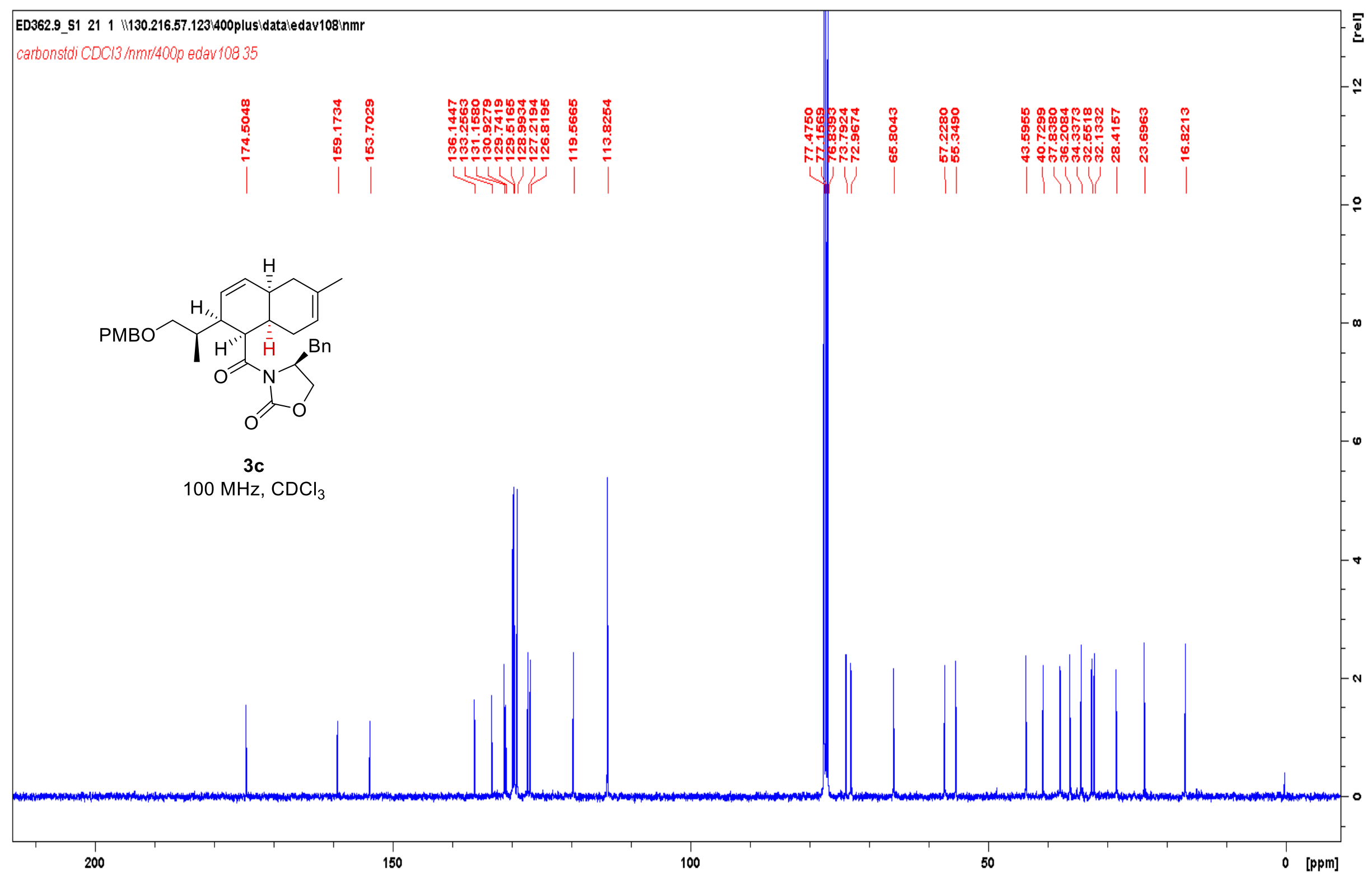




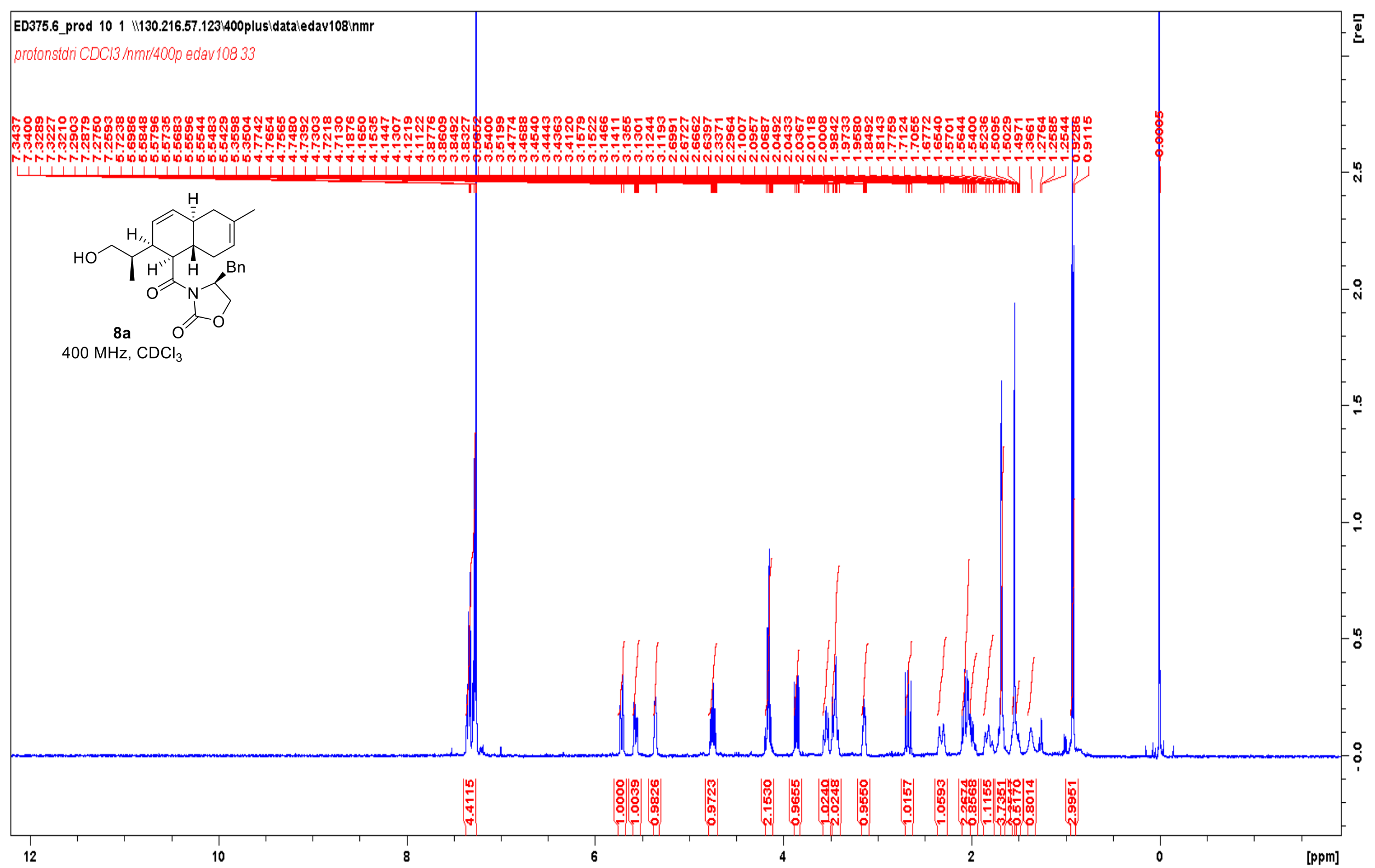


ED375.1_spot2 111 130.216.57.123\400plusidataledav108inmr

Carbonstdi CDCl3/nmr/400p edav 10835
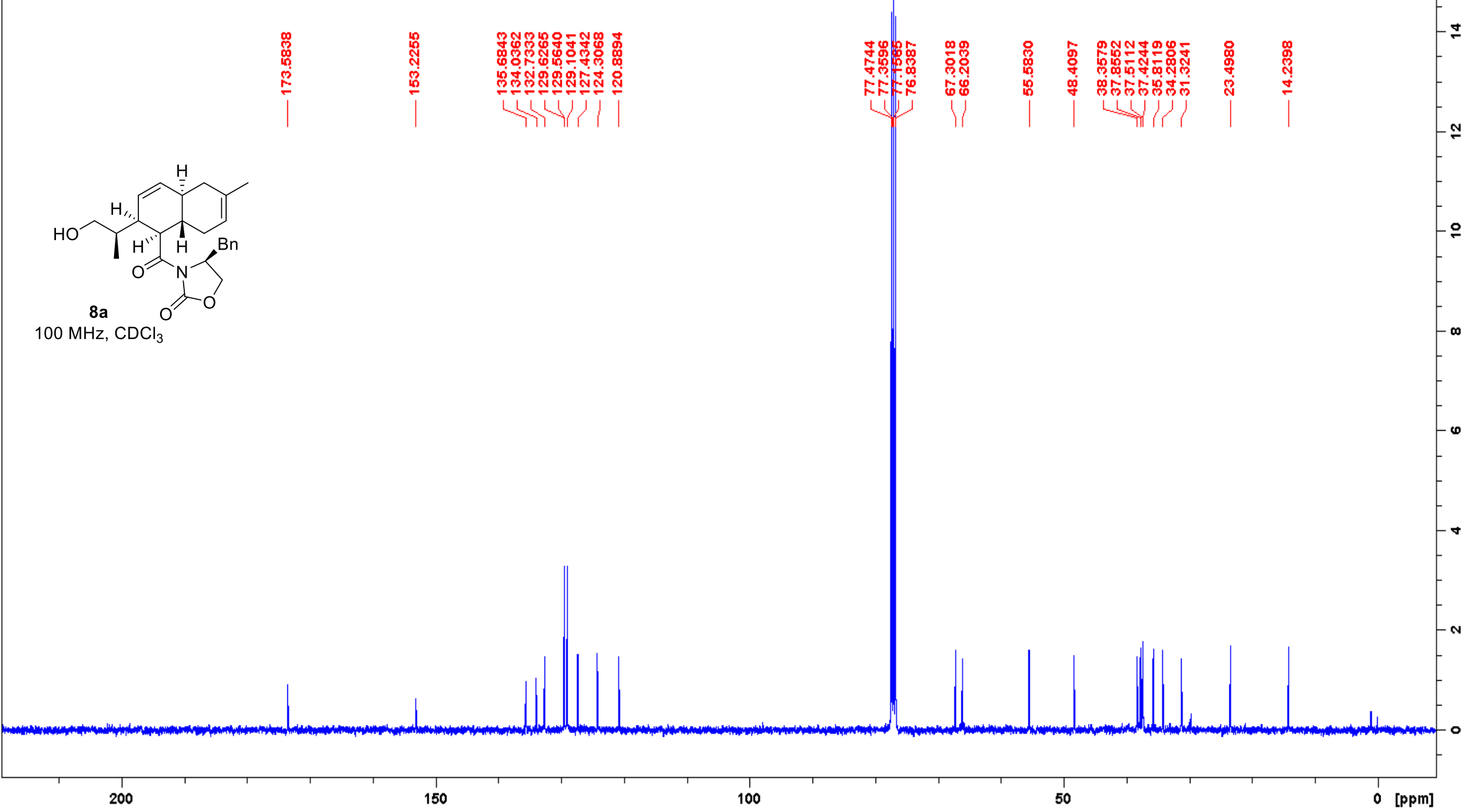

$100 \mathrm{MHz}, \mathrm{CDCl}_{3}$ 


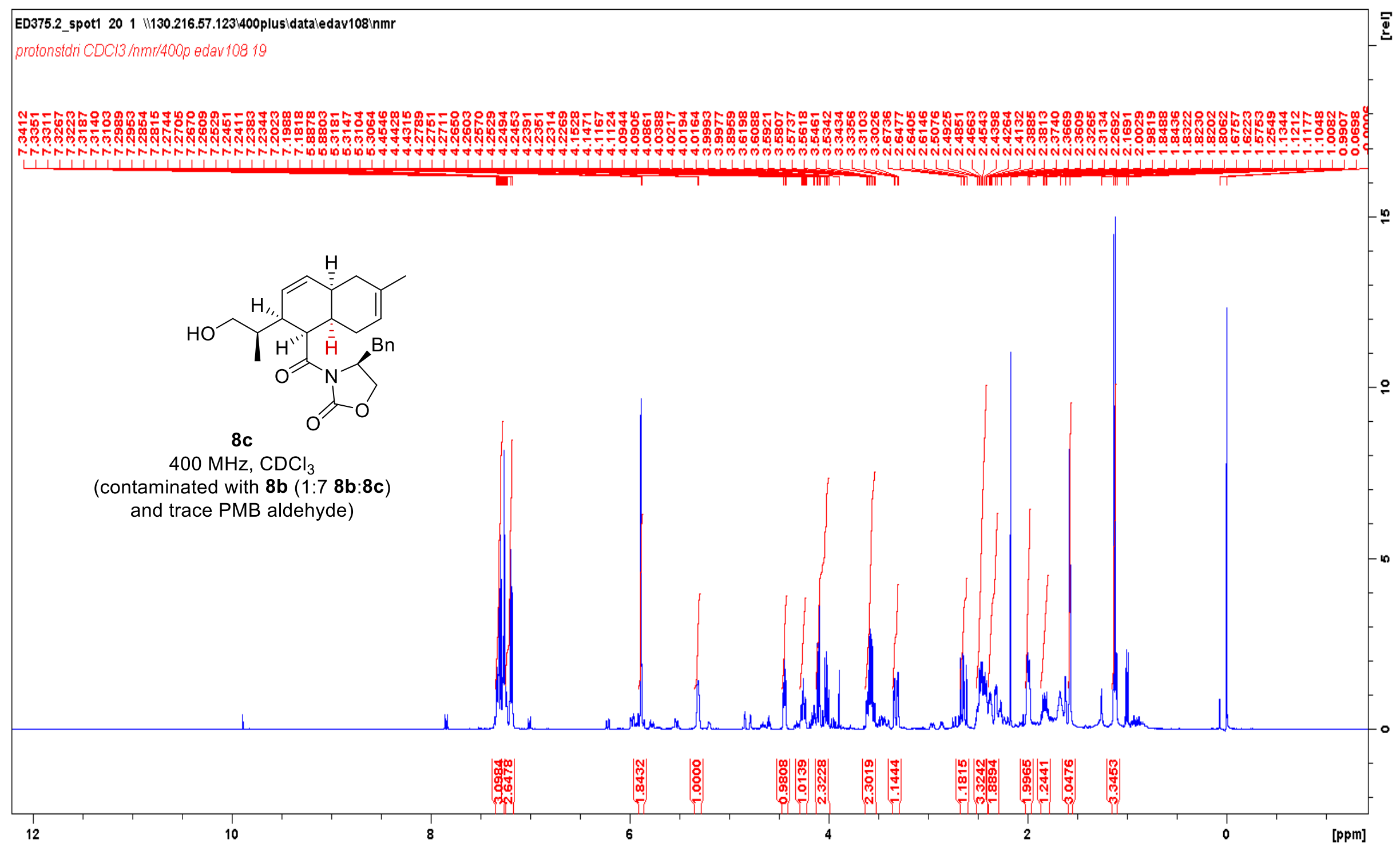




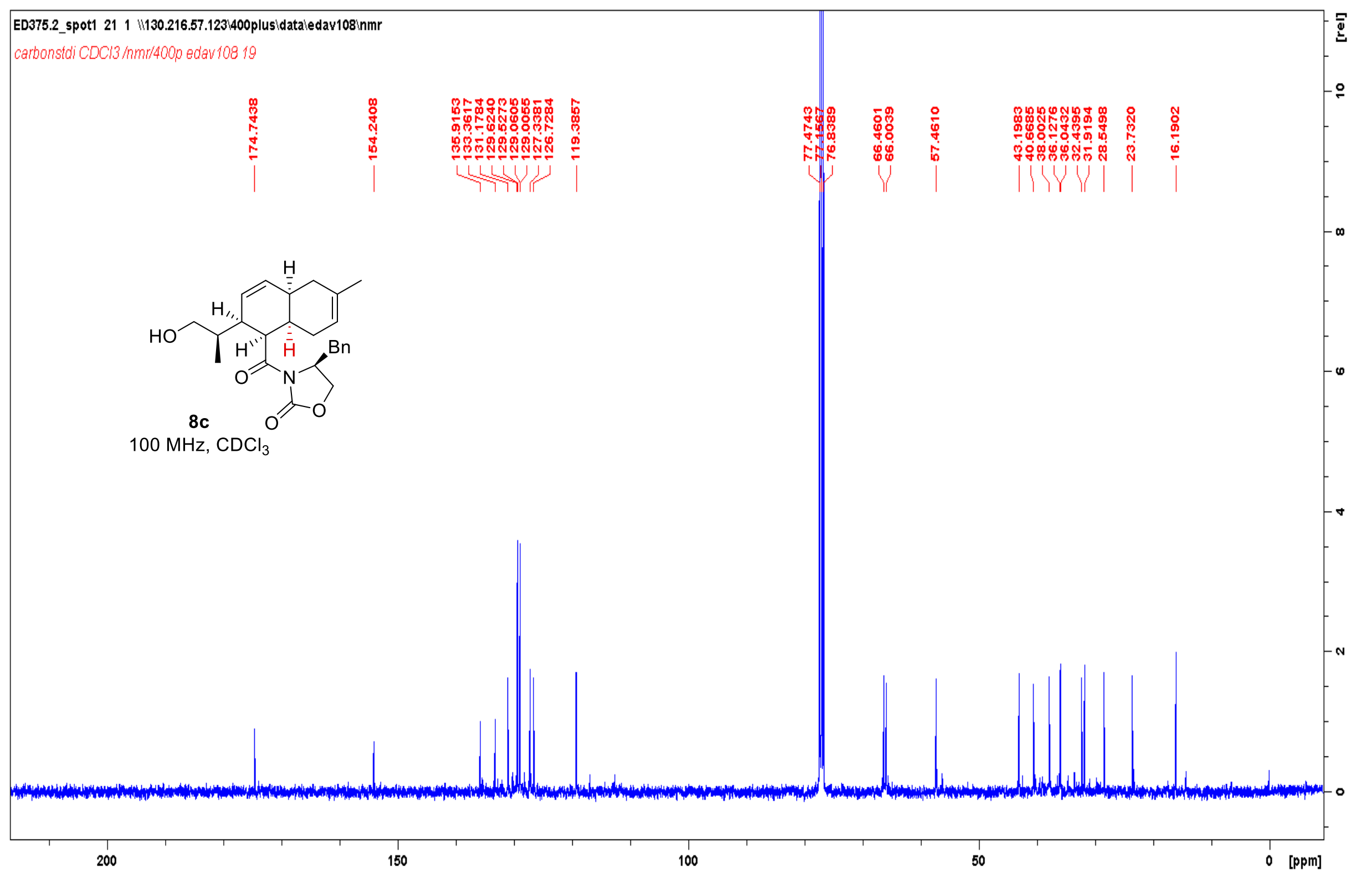




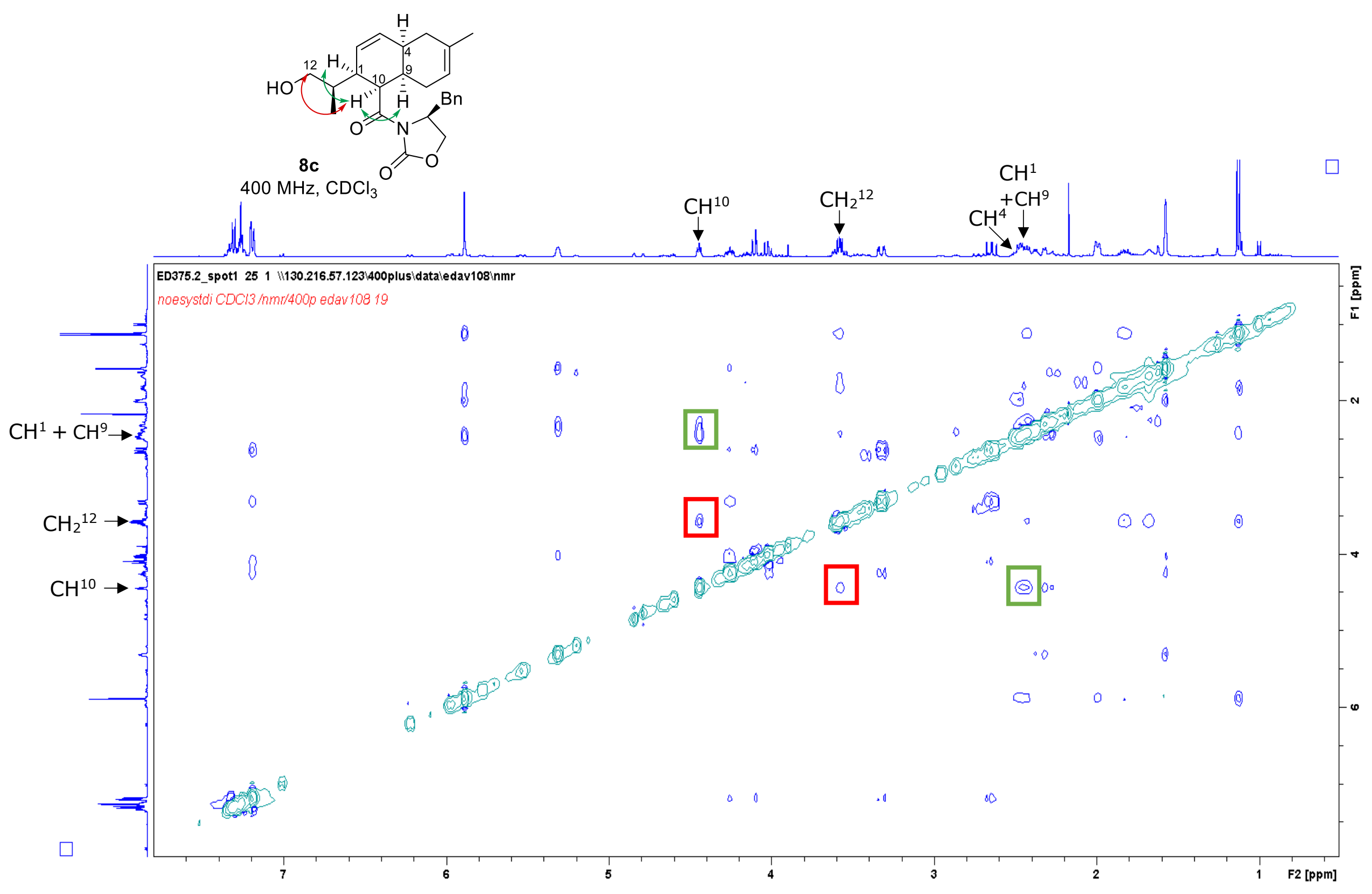




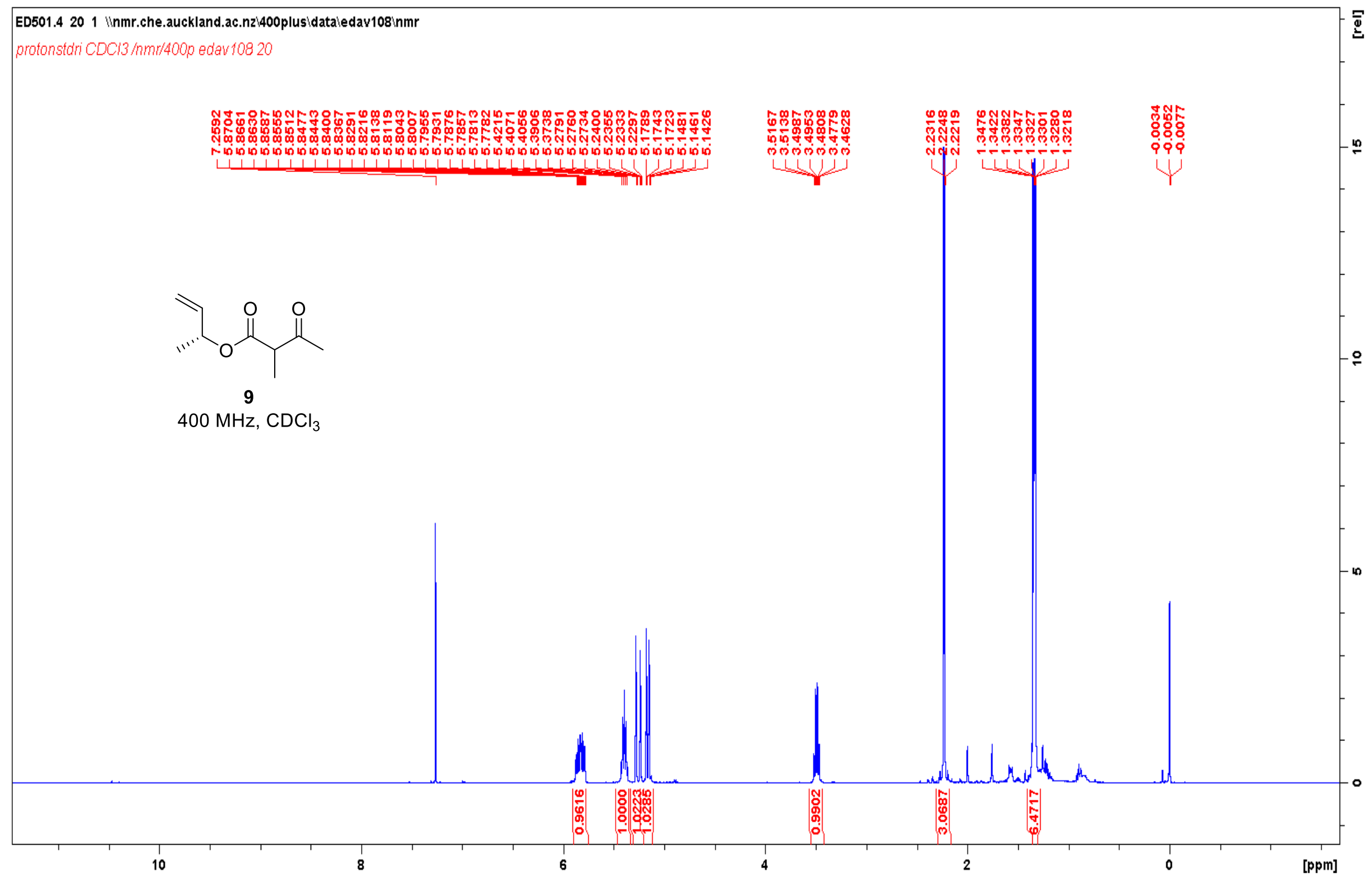




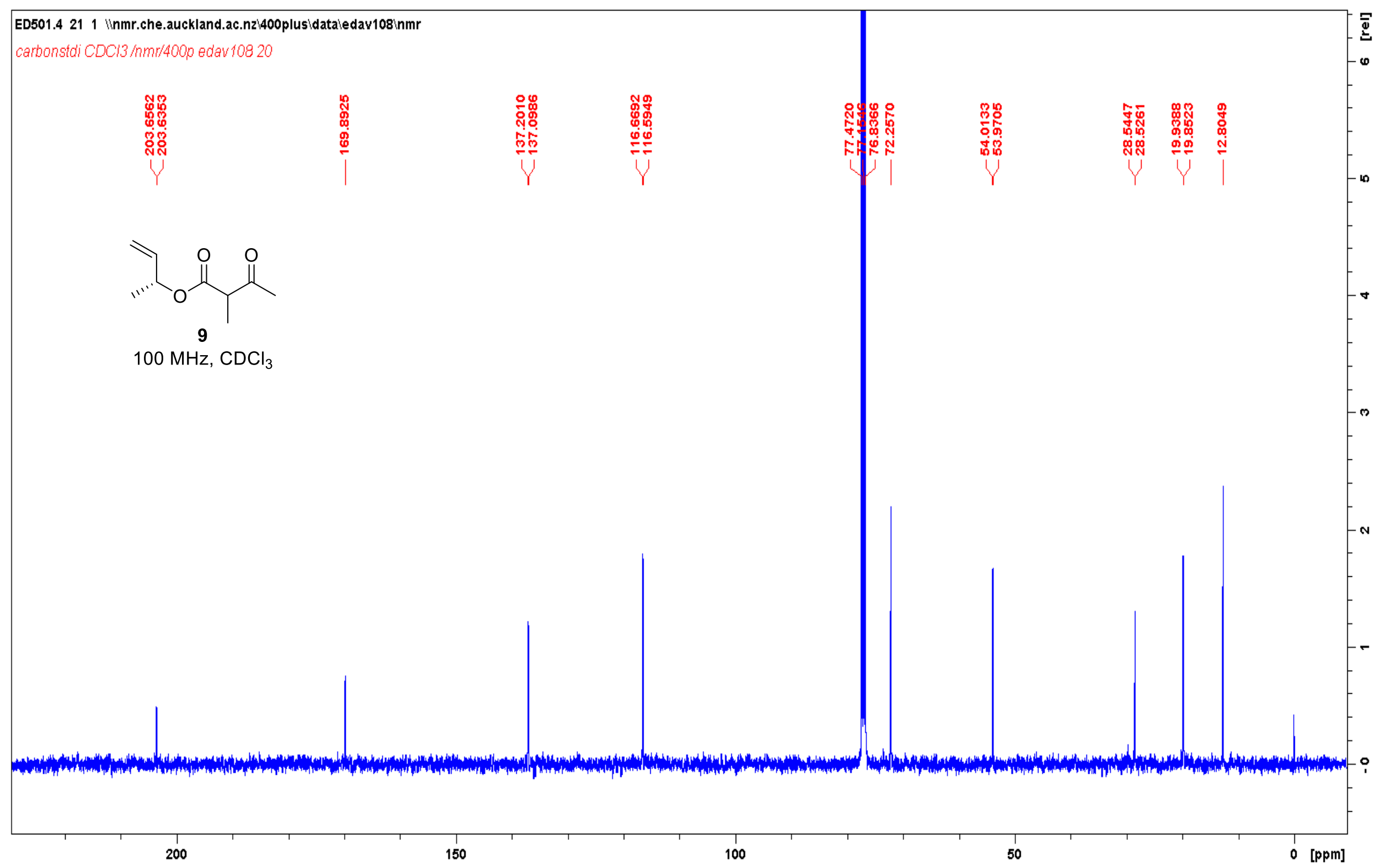




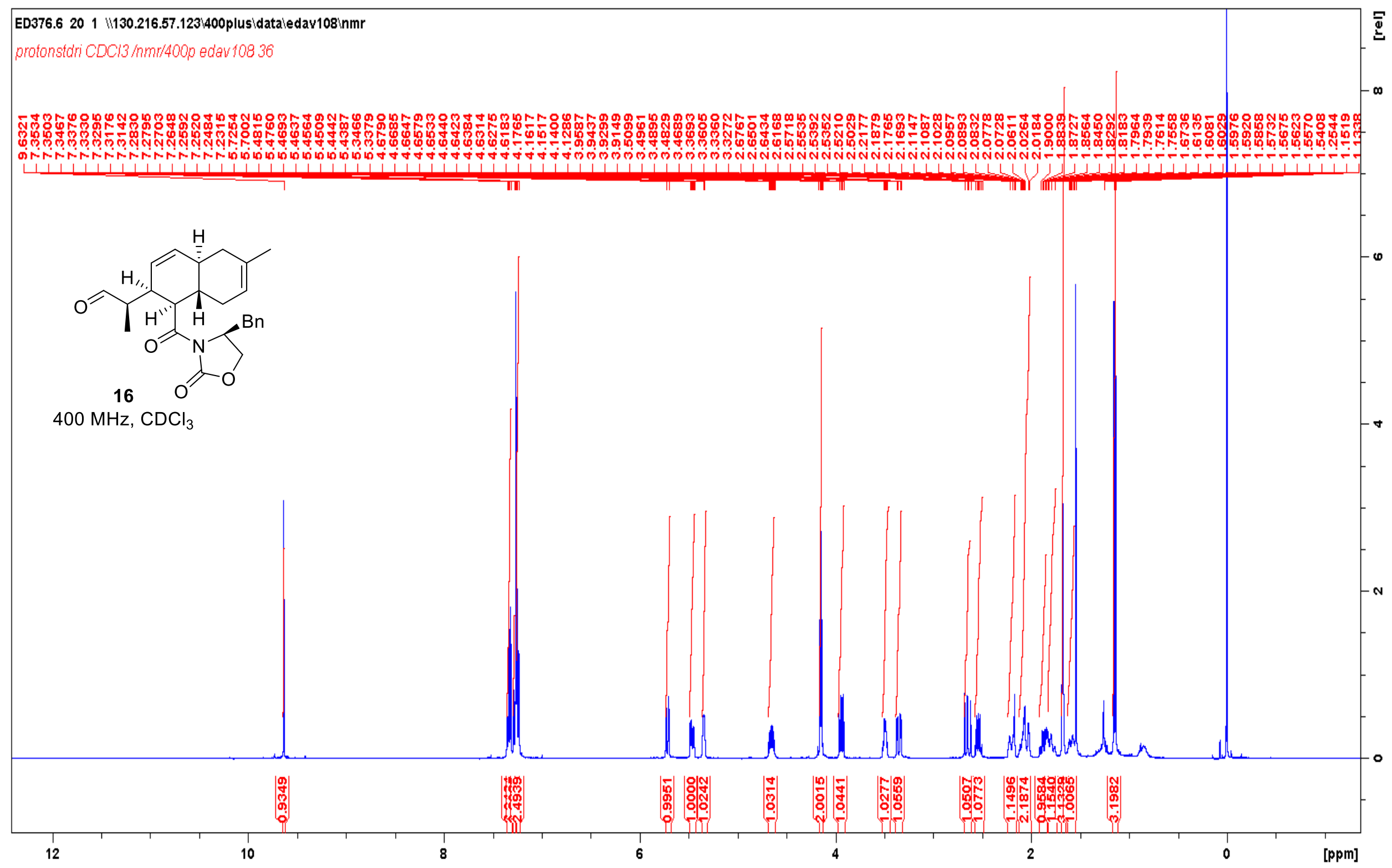




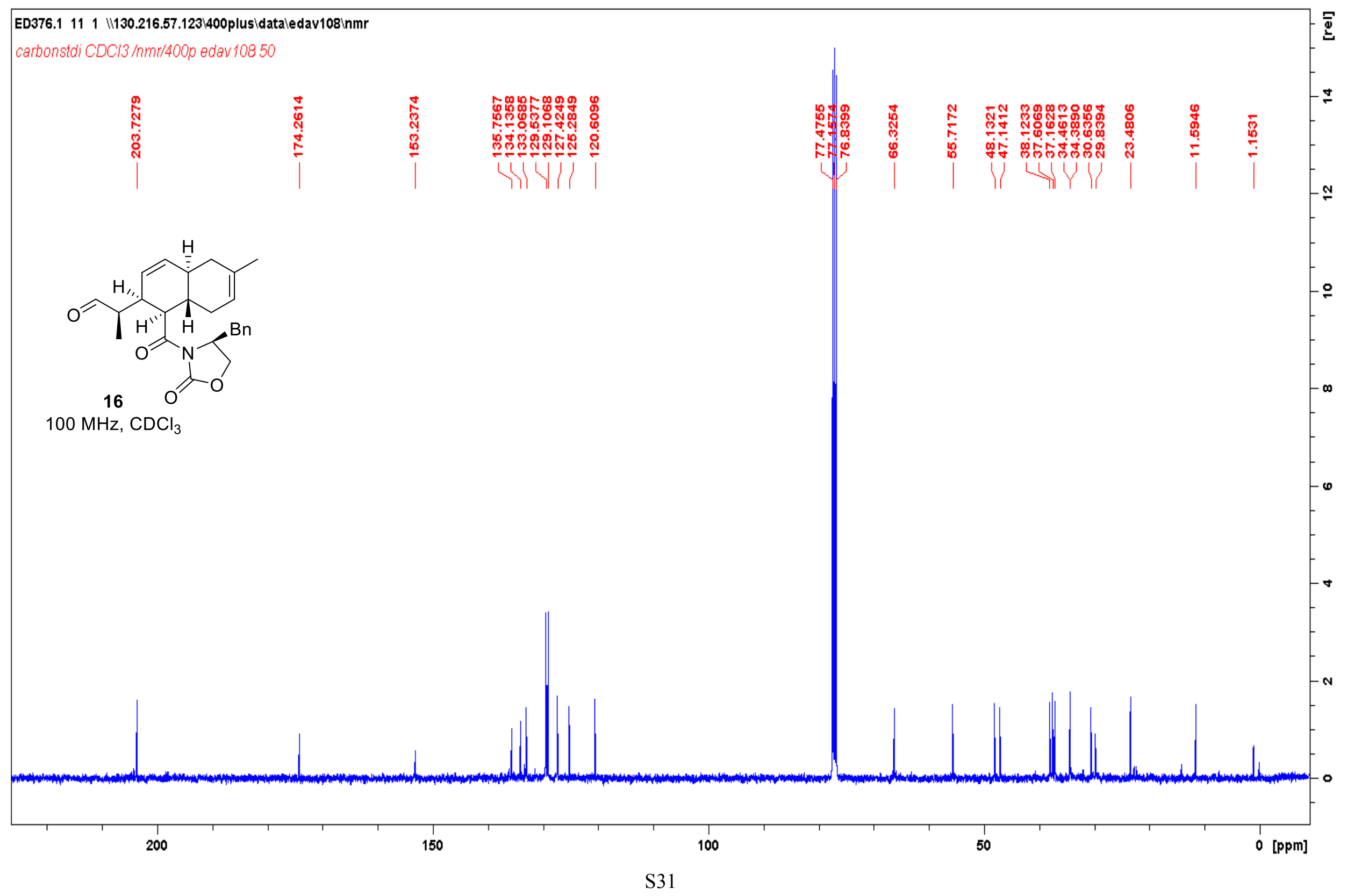




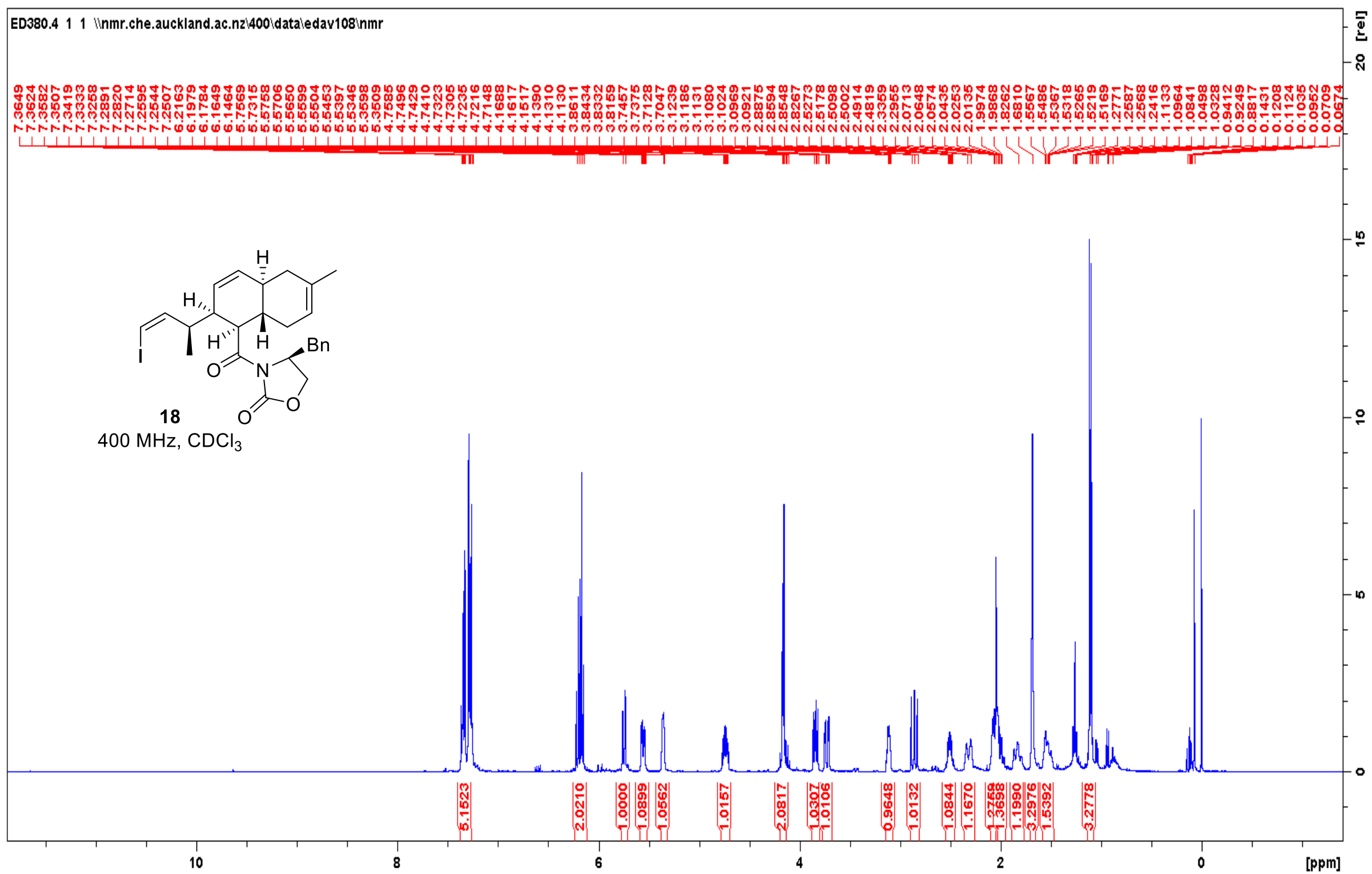




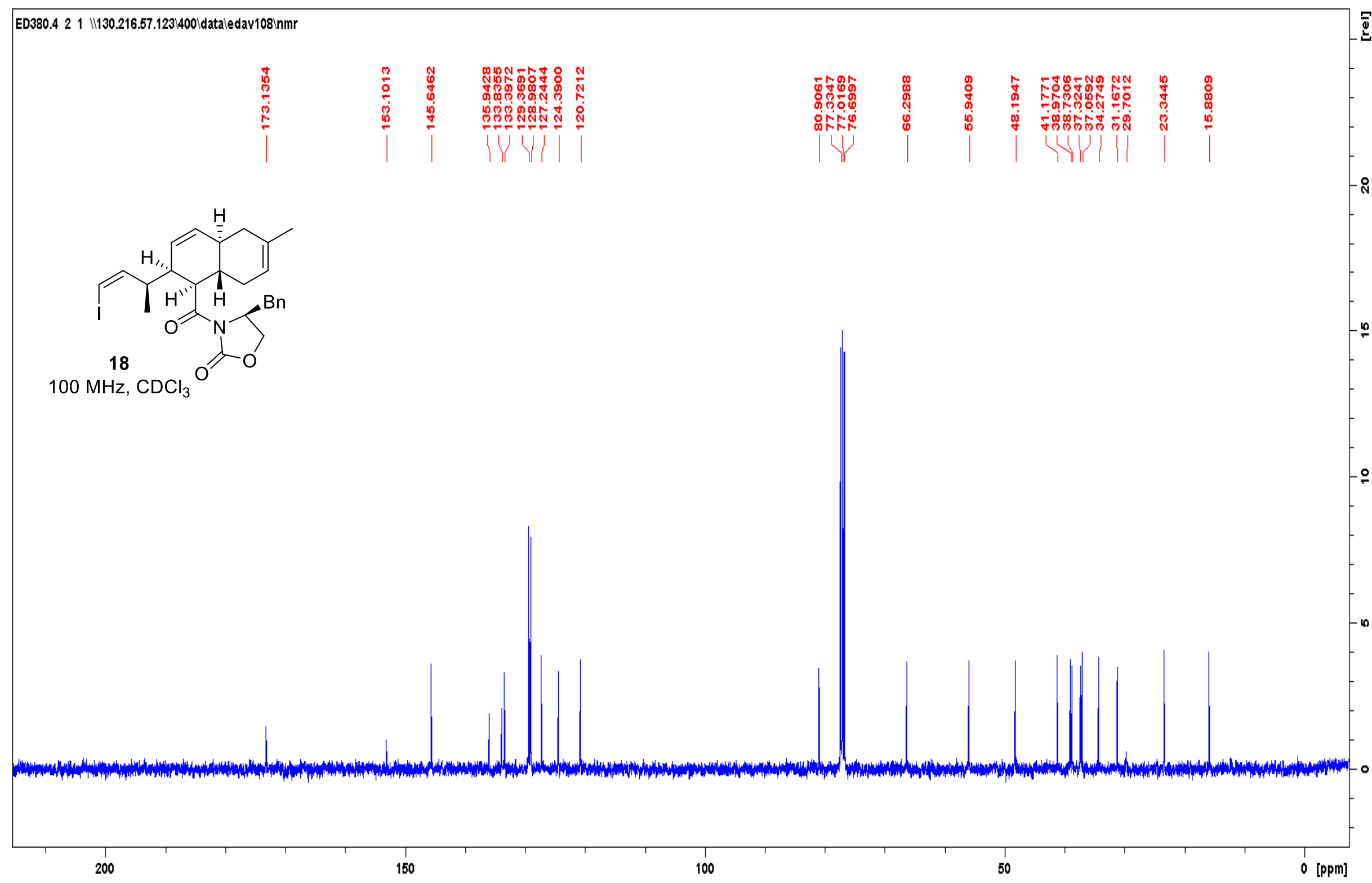




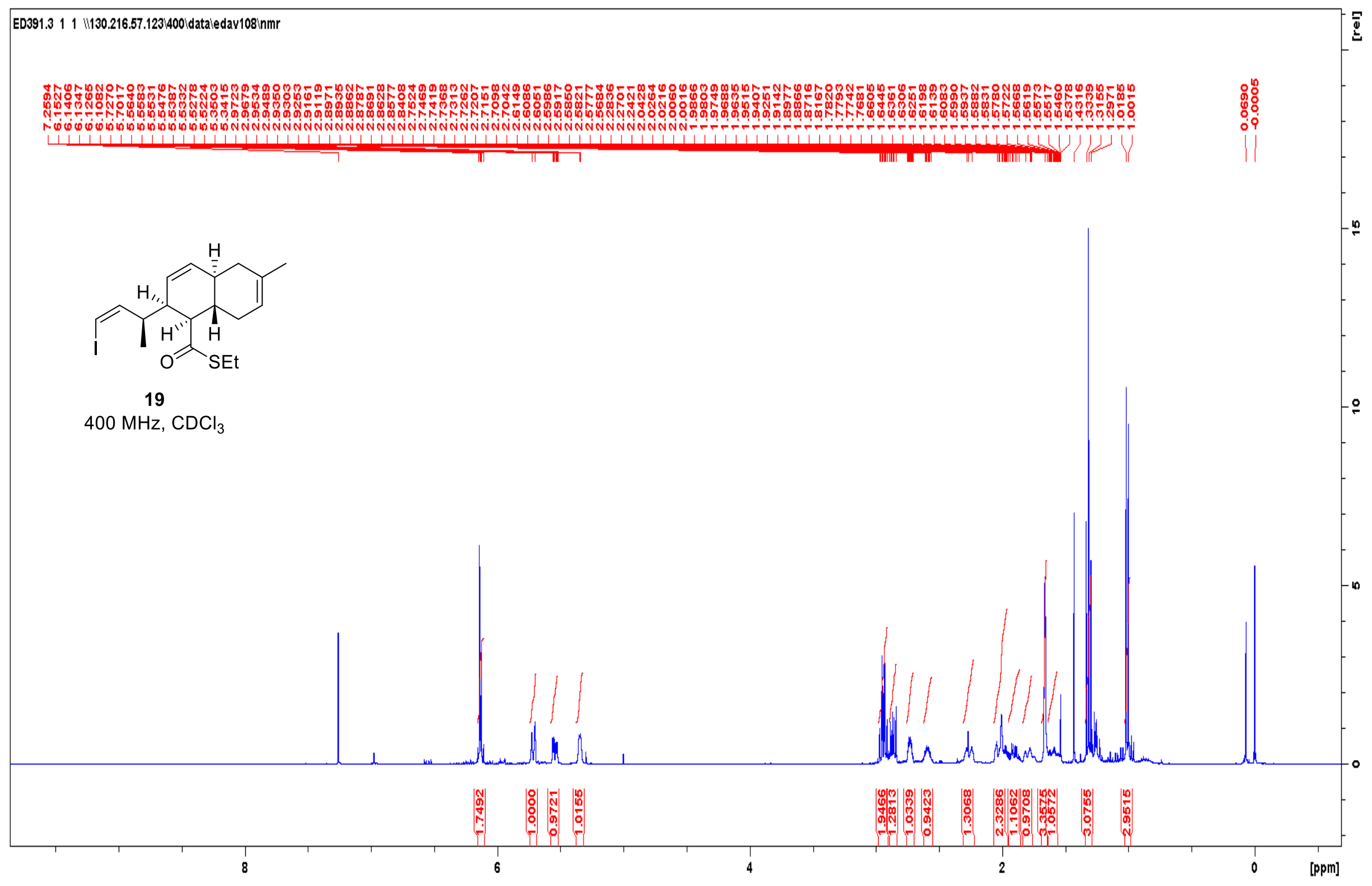




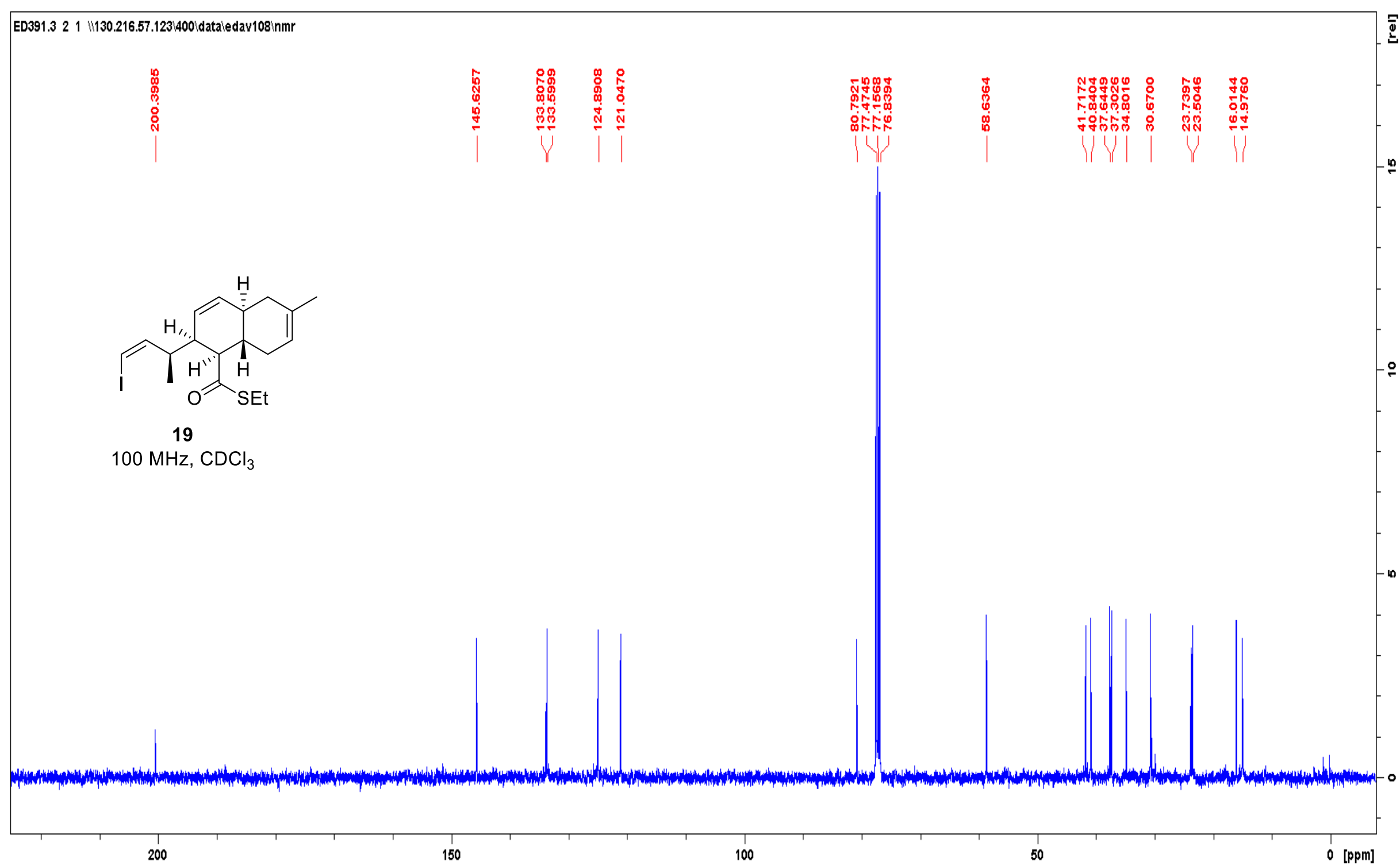




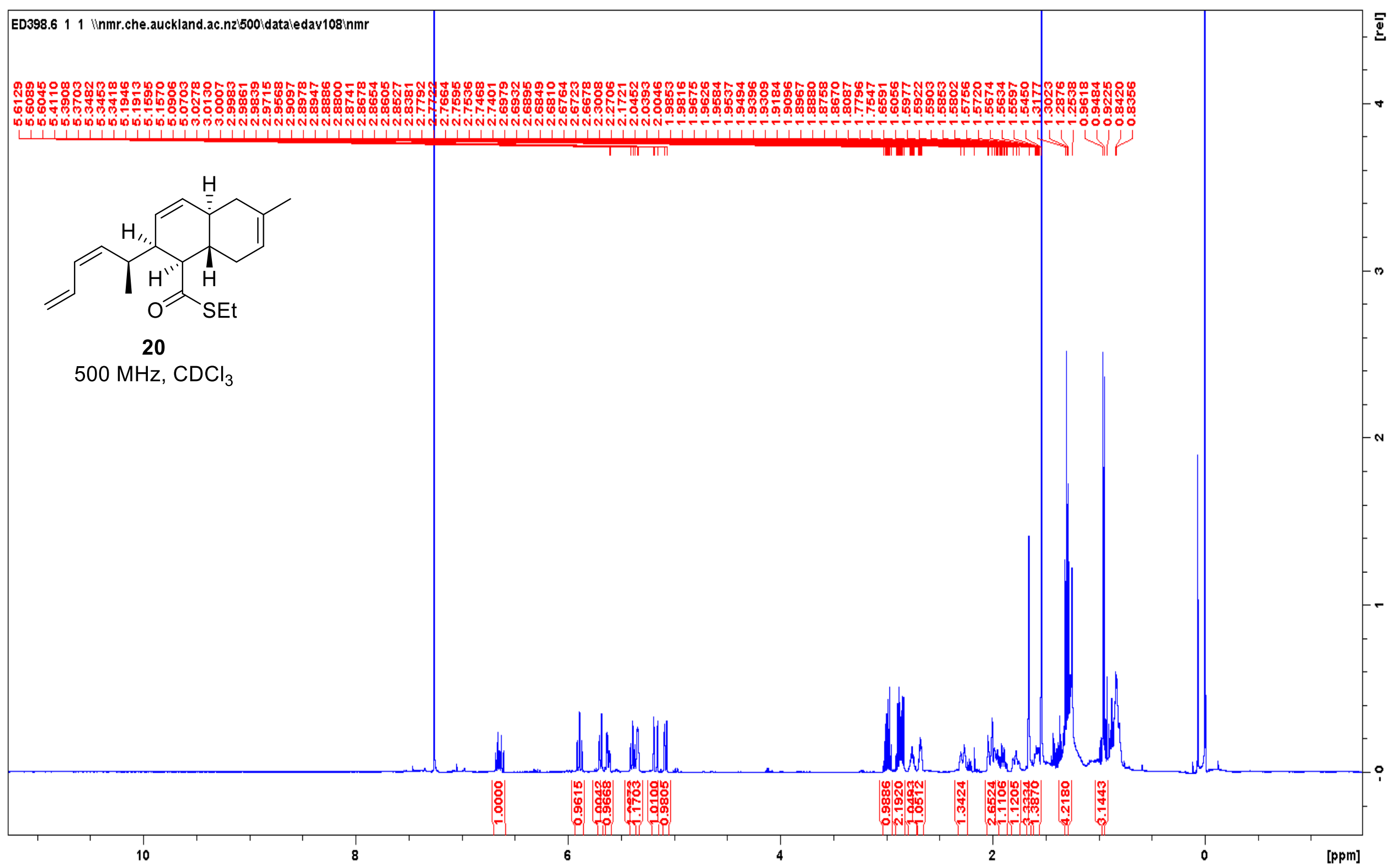




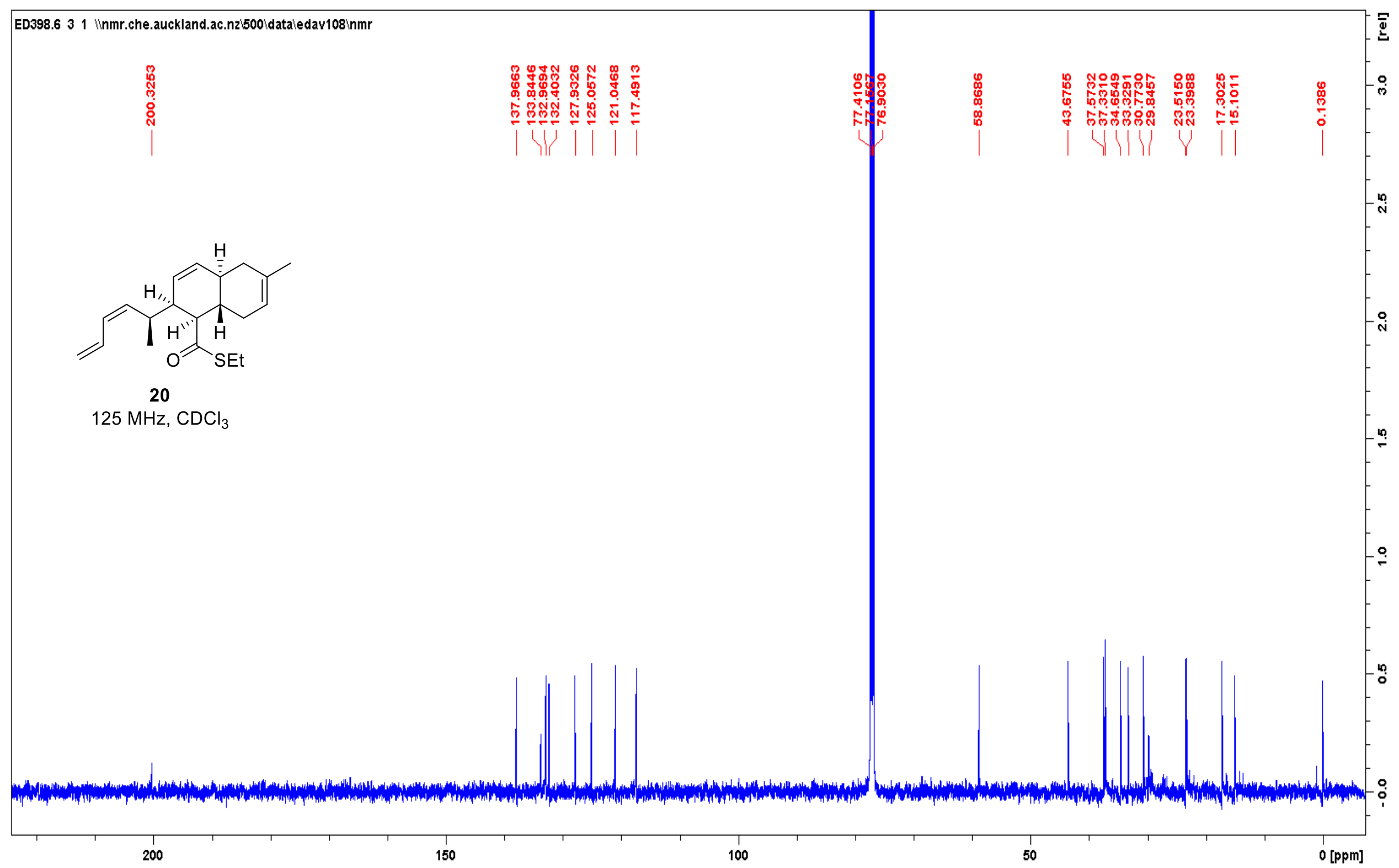




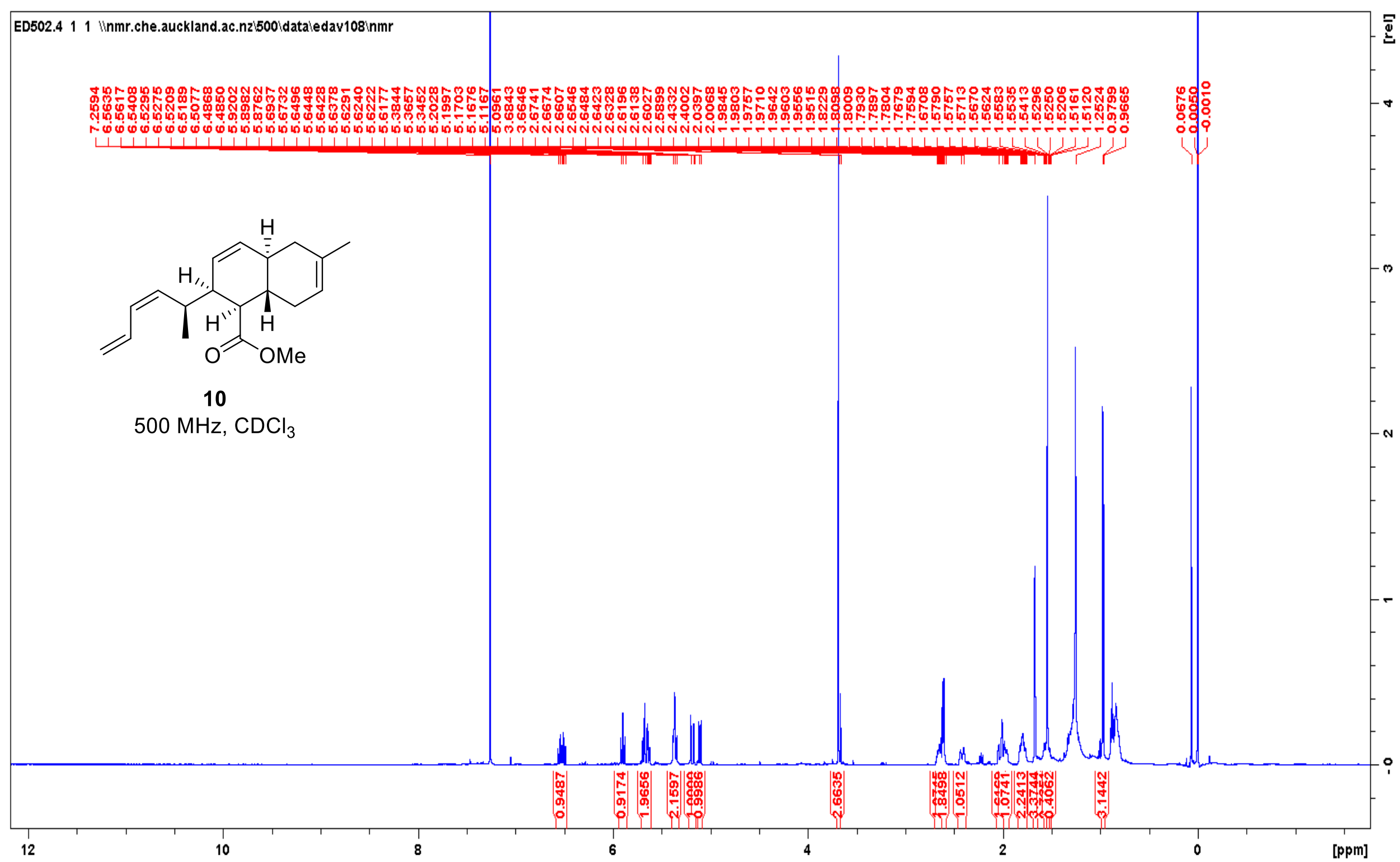




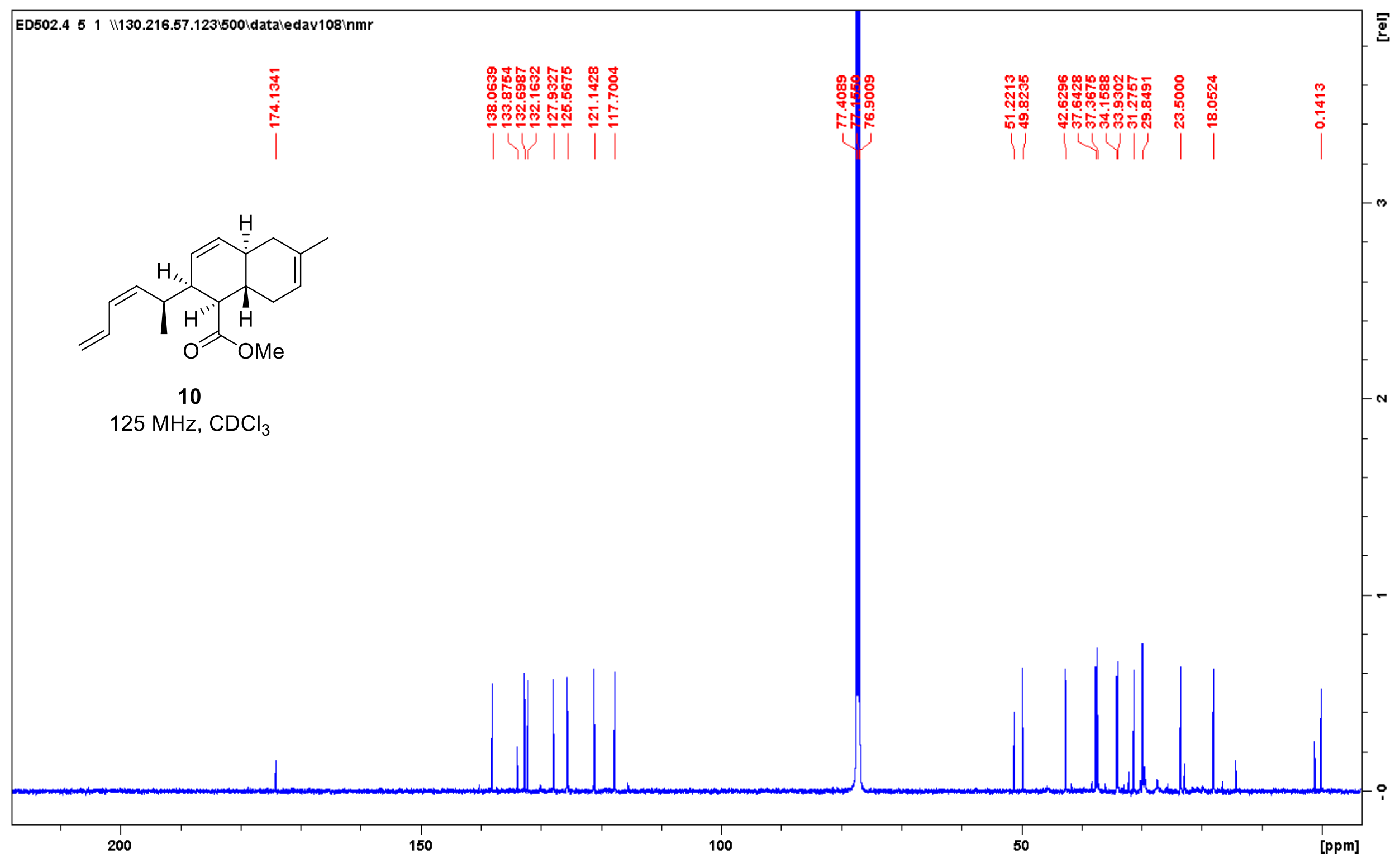




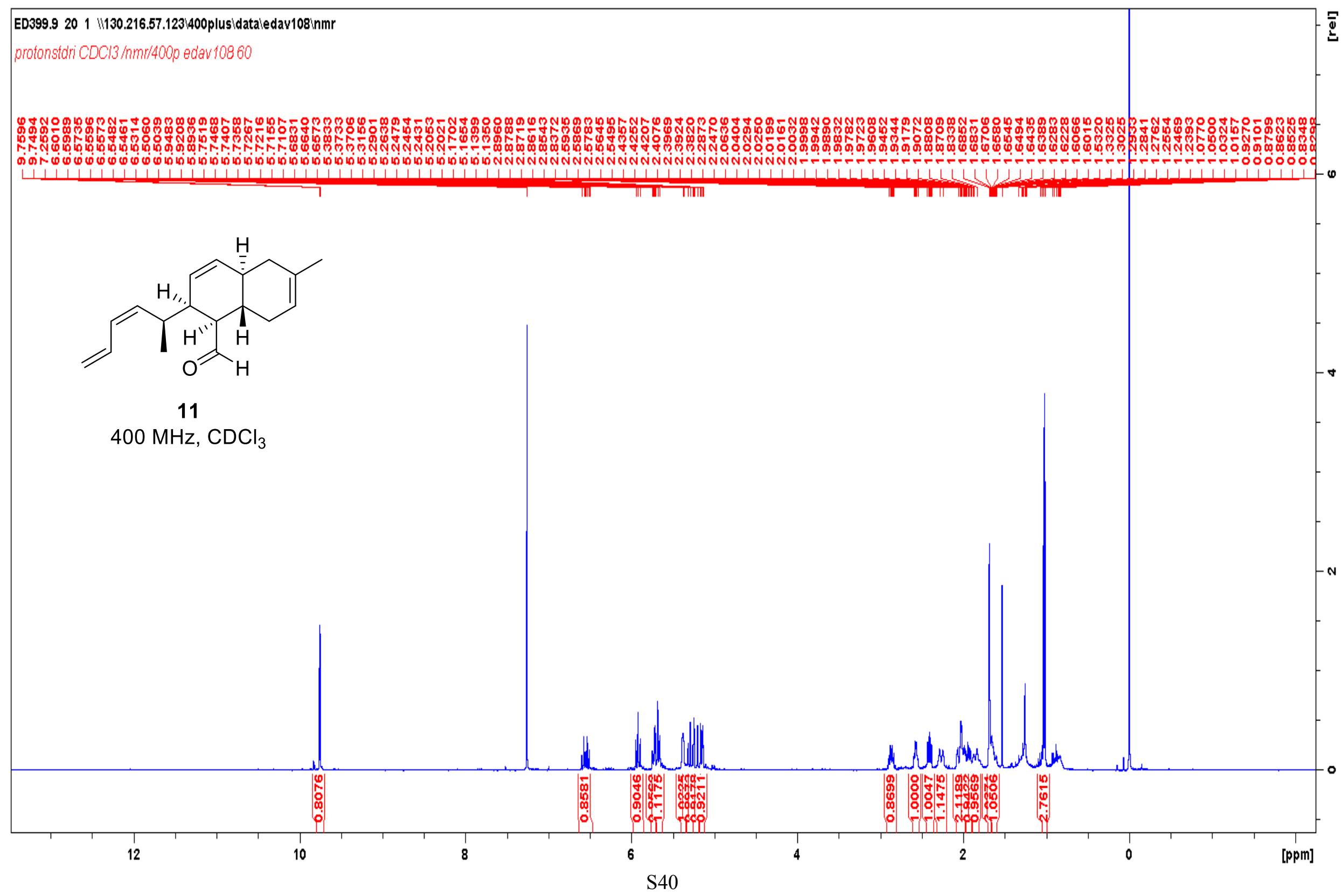




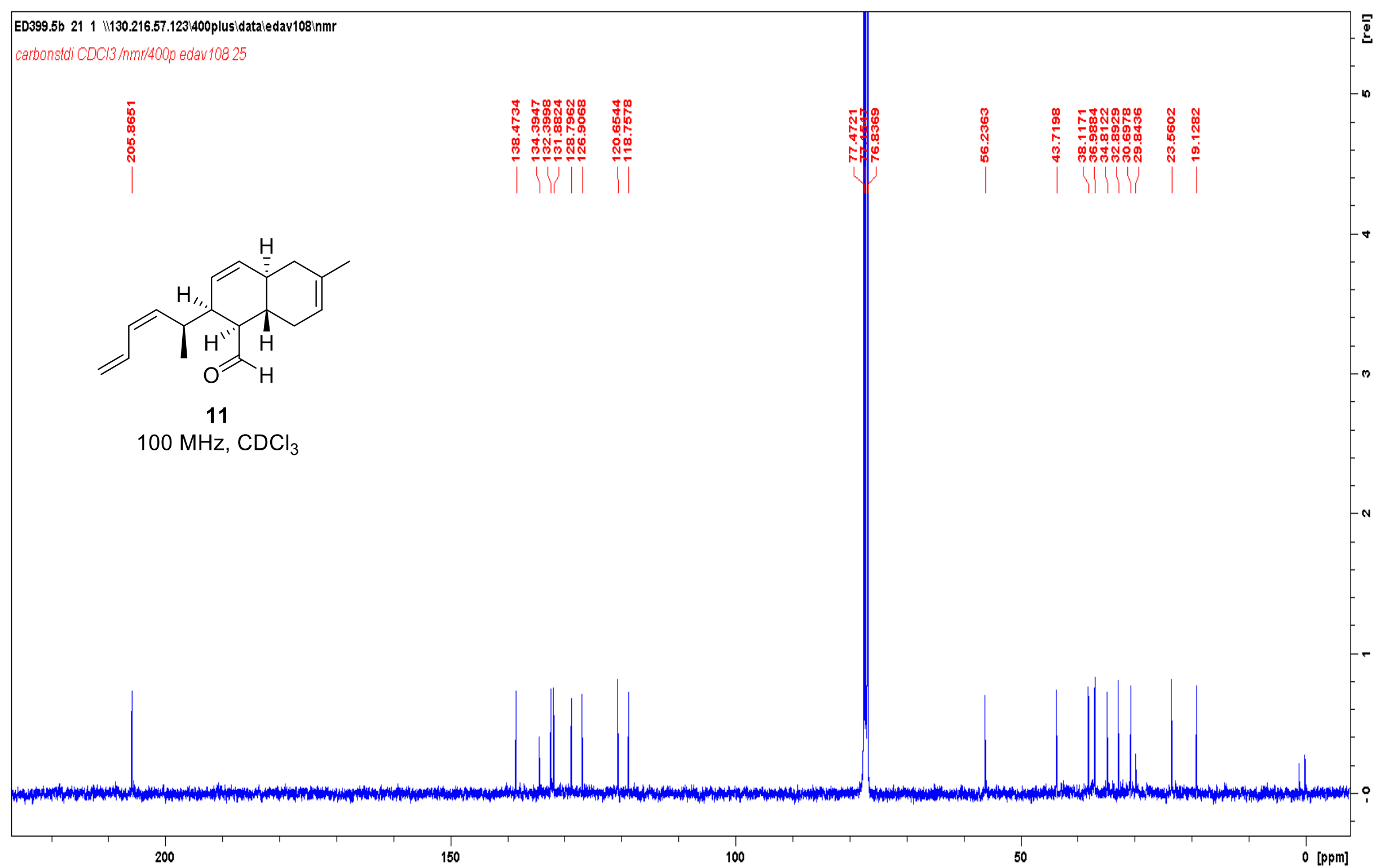




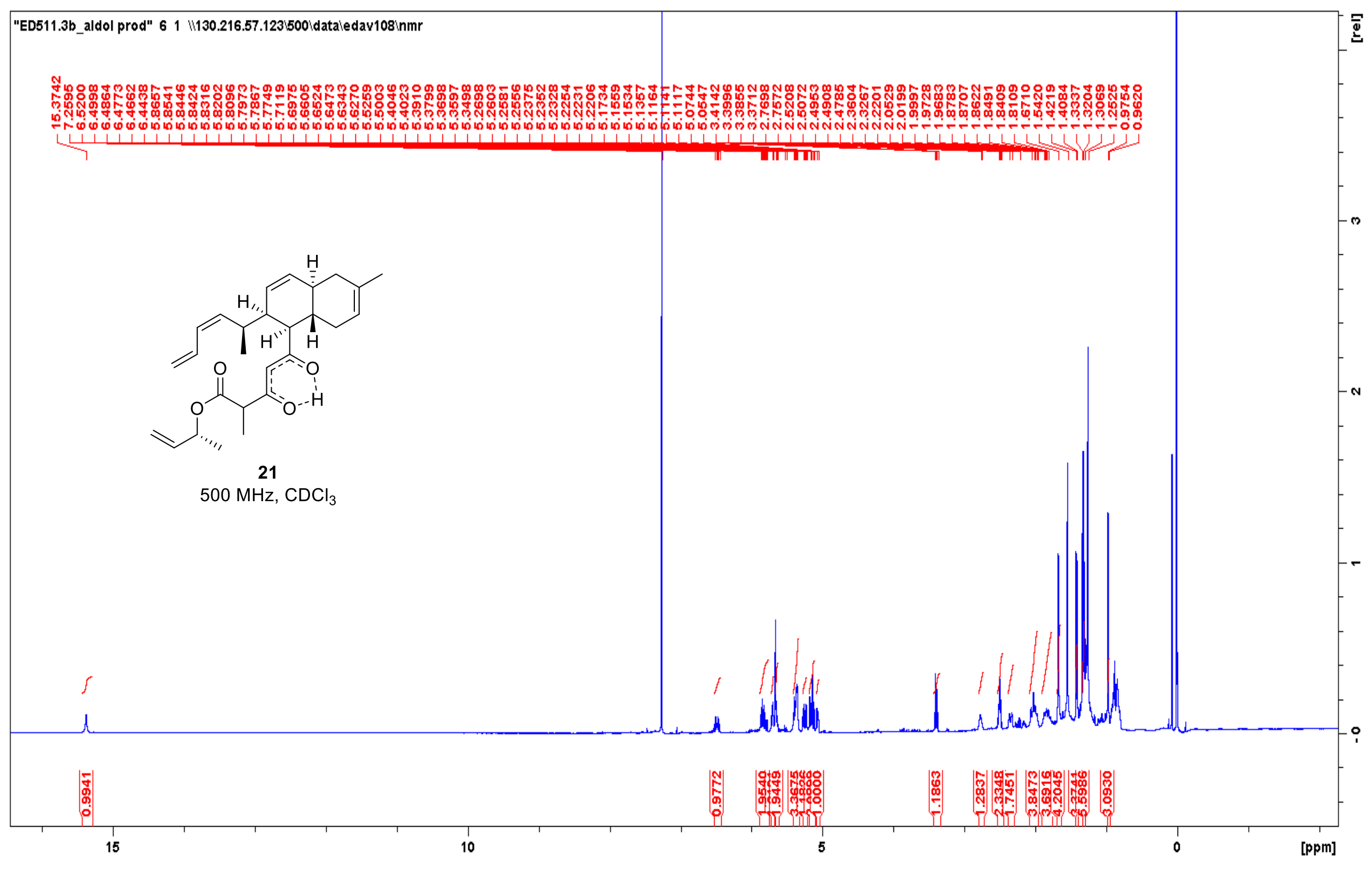




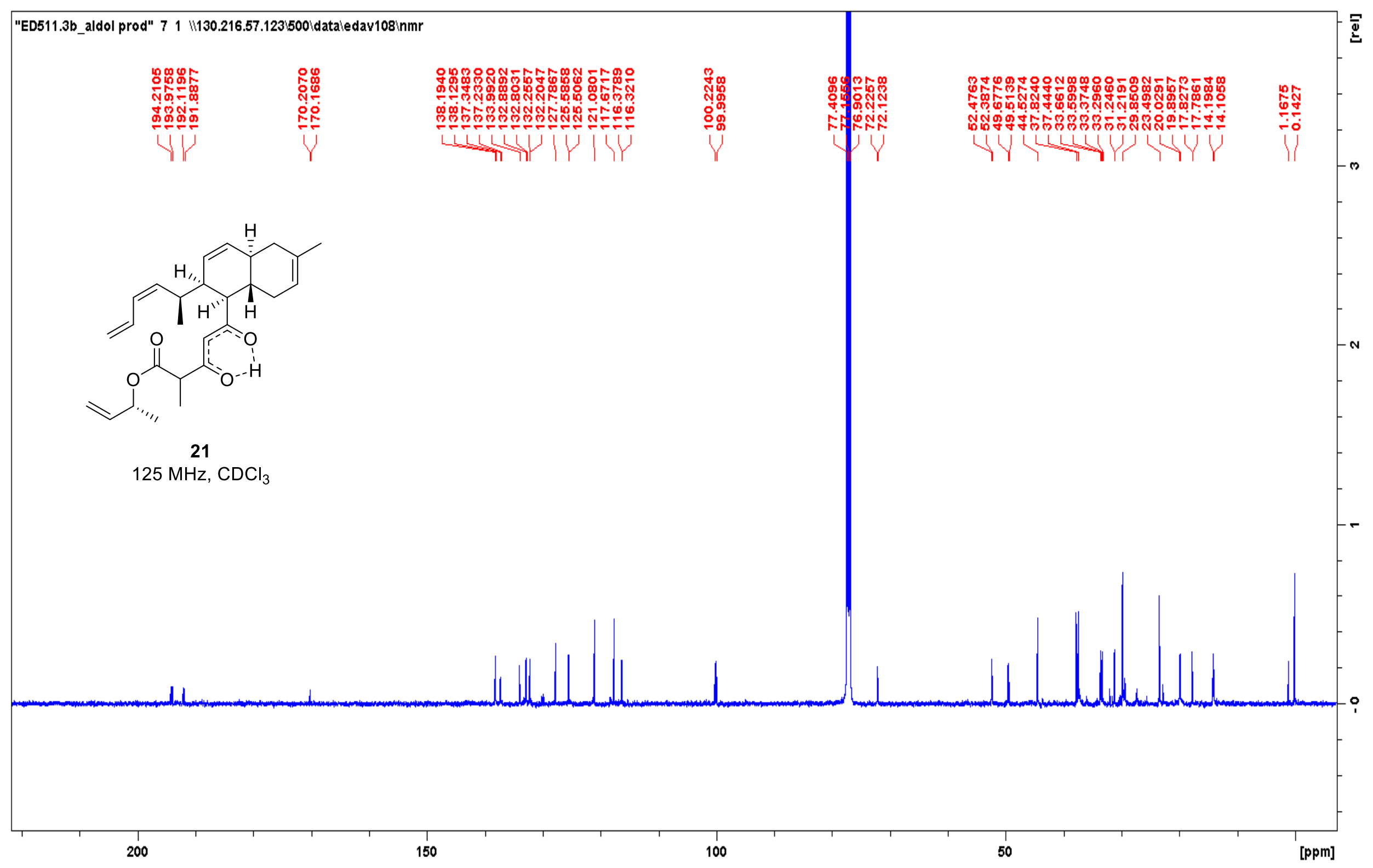




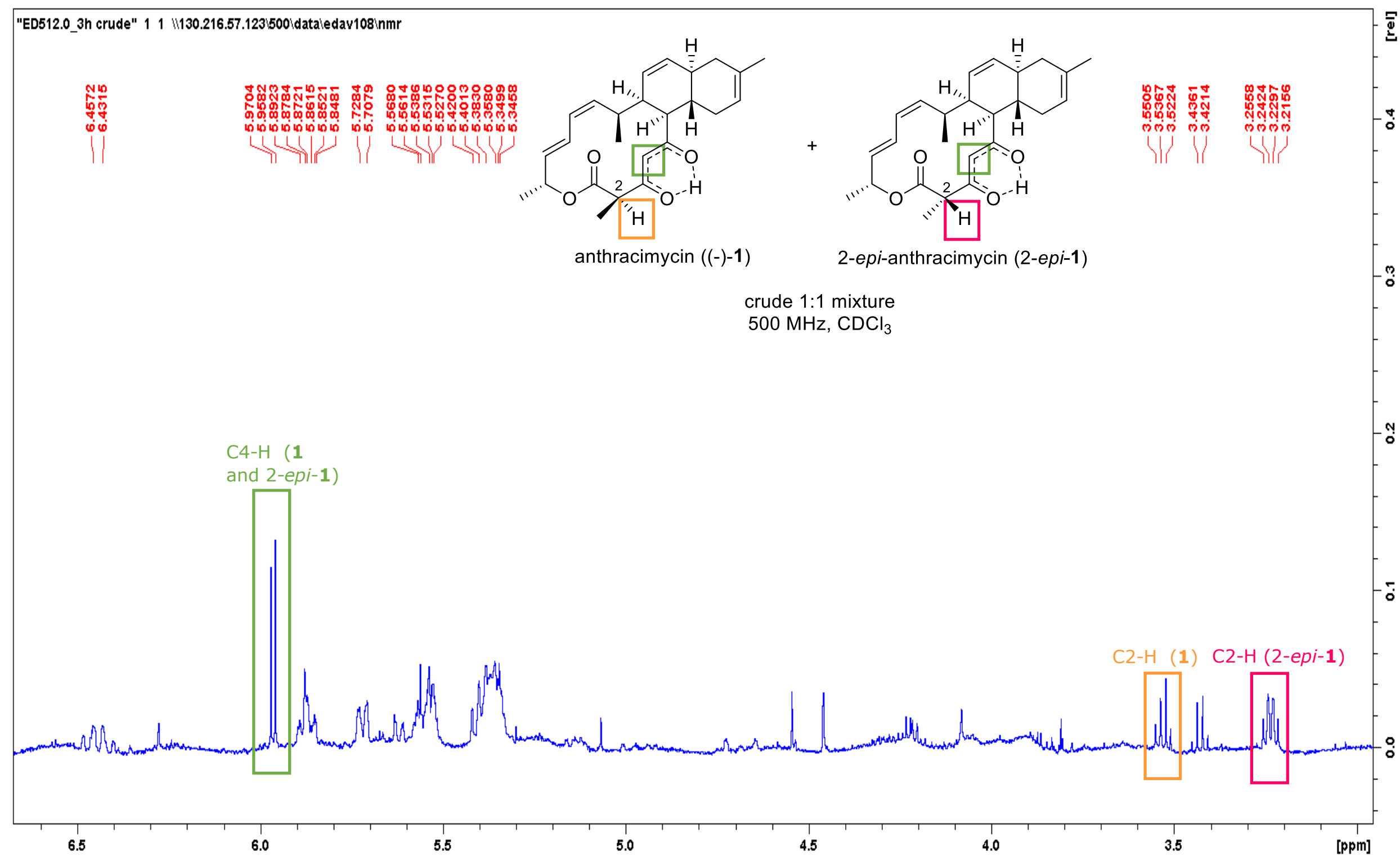




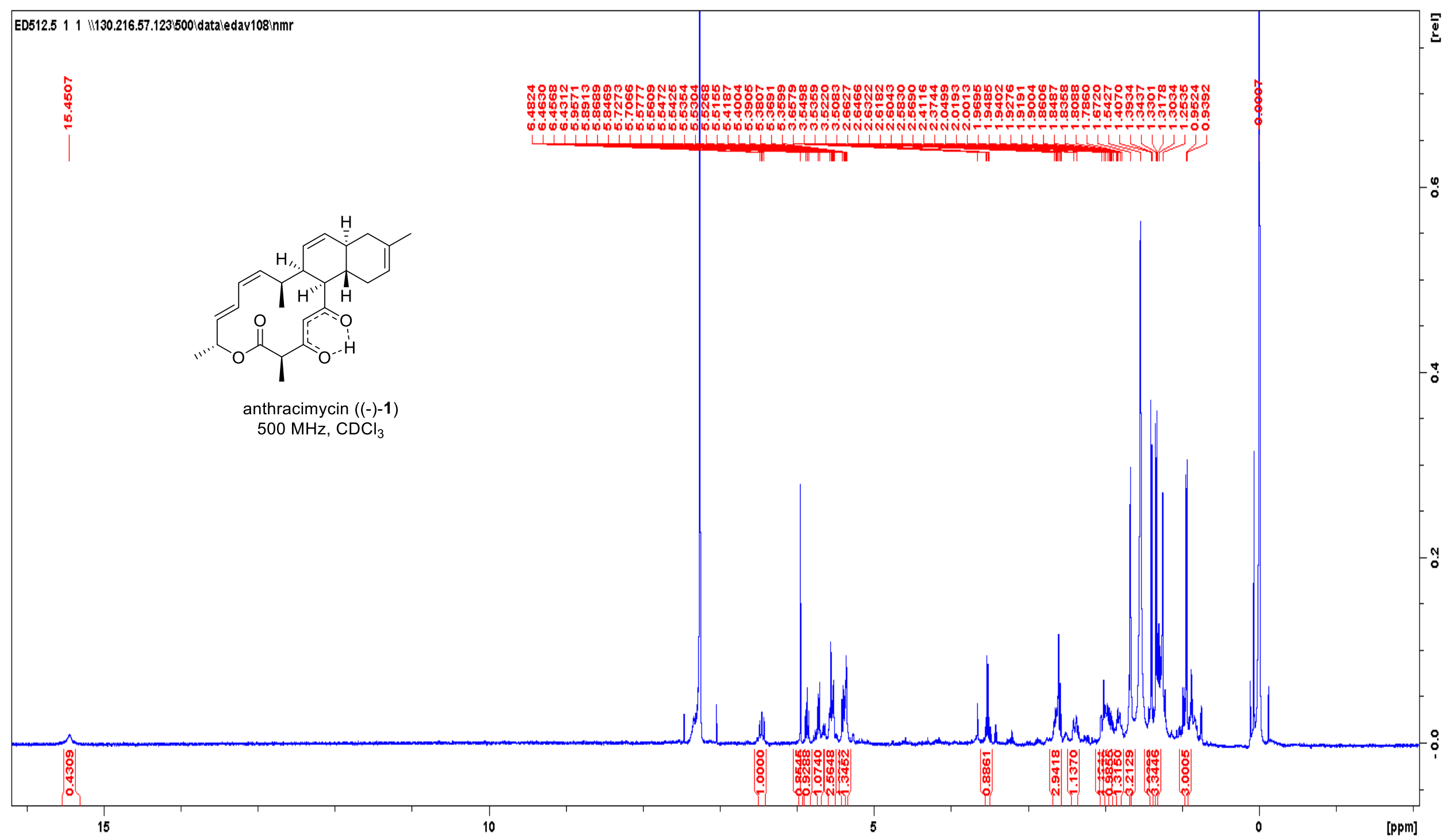




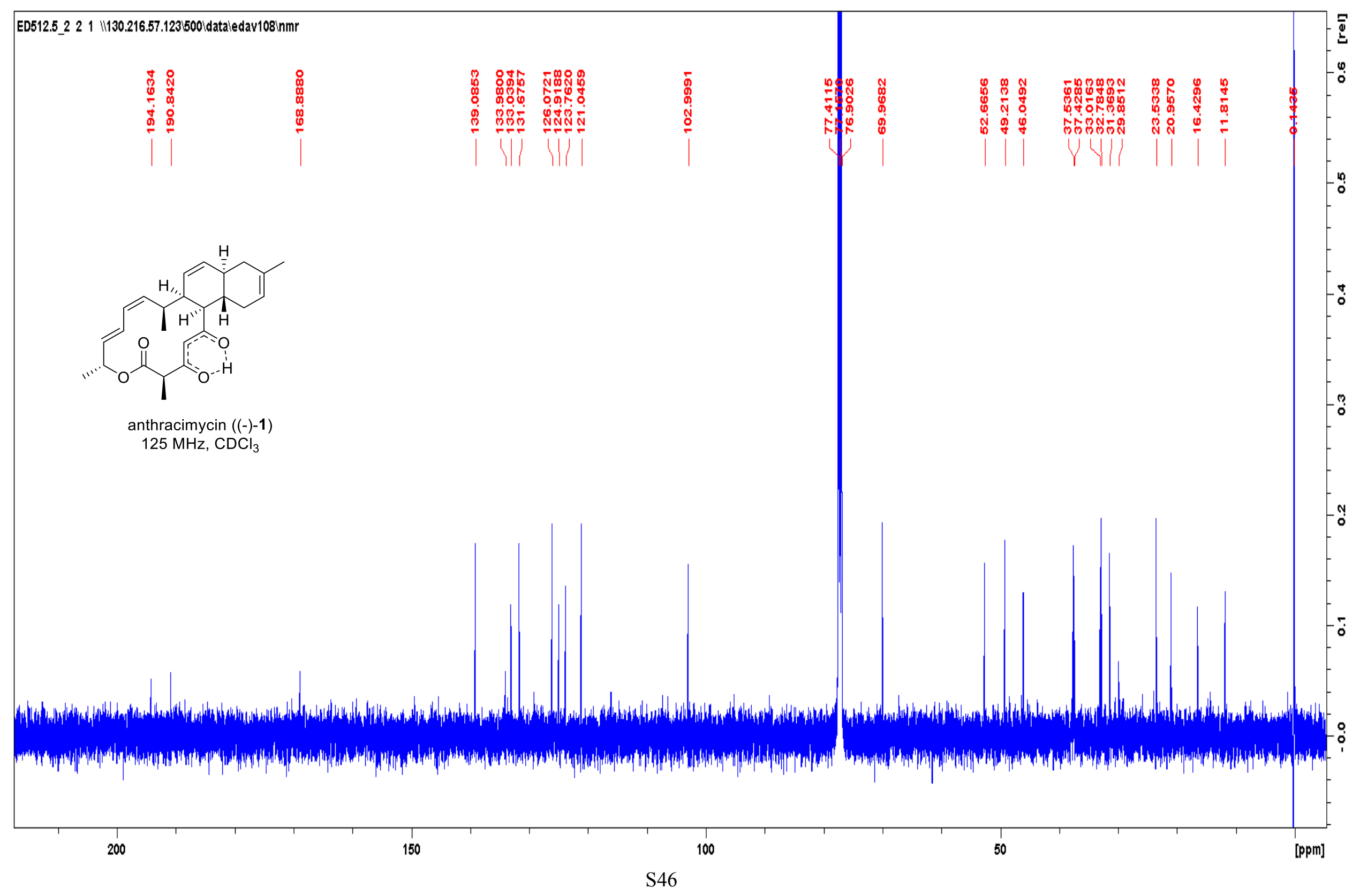

